\title{
Data report: petrography and phase analyses in lavas and dikes from Hole 1256D (ODP Leg 206 and IODP Expedition 309, East Pacific Rise) ${ }^{1}$
}

\author{
Wanja Dziony, ${ }^{2}$ Jürgen Koepke, ${ }^{2}$ and François Holtz ${ }^{2}$
}

\section{Chapter contents}

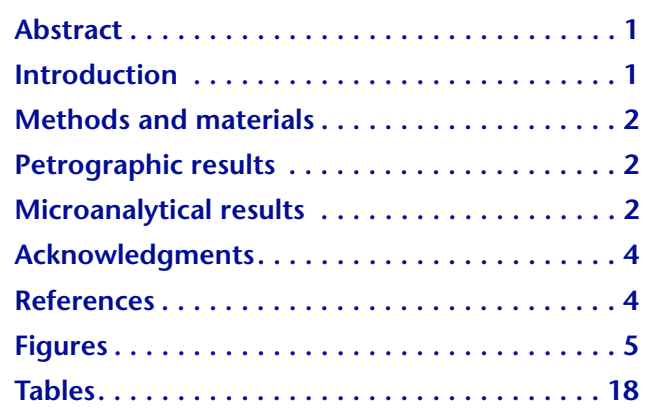

'Dziony, W., Koepke, J., and Holtz, F., 2008. Data report: petrography and phase analyses in lavas and dikes from Hole 1256D (ODP Leg 206 and IODP Expedition 309, East Pacific Rise). In Teagle, D.A.H., Alt, J.C., Umino, S., Miyashita, S., Banerjee, N.R., Wilson, D.S., and the Expedition 309/312 Scientists, Proc. IODP, 309/312: Washington, DC (Integrated Ocean Drilling Program Management International, Inc.).

doi:10.2204/iodp.proc.309312.201.2008

IInstitut fuer Mineralogie, Leibniz University Hannover, Callinstr. 3, 30167 Hannover, Germany. Correspondence author:

w.dziony@mineralogie.uni-hannover.de

\section{Abstract}

We investigated 56 basalts drilled in Hole 1256D (equatorial East Pacific Rise) during Ocean Drilling Program Leg 206 and Integrated Ocean Drilling Program Expedition 309. Primary magmatic phases, pyroxenes, plagioclases, and Fe-Ti oxides were analyzed in 16 selected samples by electron microprobe analysis. Both clinopyroxene and plagioclase show strong zoning effects, especially in the outermost rims. Many clinopyroxenes show marked zoning, which is in part developed as sector zoning. Some clinopyroxenes bear cores of relict pigeonites, which show slightly higher $\mathrm{Mg \#}\left(100\right.$ * molar $\mathrm{MgO} /\left[\mathrm{MgO}+\mathrm{FeO}^{\text {tot }}\right]$, where $\mathrm{FeO}^{\text {tot }}=$ total $\mathrm{FeO}$ ) than the host crystals. Plagioclase shows a broad scattering in composition, often combined with zoning. We observed a slight trend toward more evolved compositions of mineral phases in the sheeted dikes. Only 1 of the 16 samples bears coexisting magnetite and ilmenite (from the uppermost volcanic unit, the lava pond). By applying the magnetite-ilmenite thermooxybarometer, we calculated an oxygen fugacity corresponding to $\triangle \mathrm{FMQ}$ of -0.4 ( $0.4 \mathrm{log}$ units below the oxygen fugacity of the fayalite-magnetite-quartz buffer).

\section{Introduction}

Integrated Ocean Drilling Program (IODP) Hole $1256 \mathrm{D}\left(6.736^{\circ} \mathrm{N}\right.$, $91.934^{\circ} \mathrm{W}$ ), drilled during Ocean Drilling Program (ODP) Leg 206 and IODP Expedition 309/312 (Wilson et al., 2006) is located on 15 Ma oceanic crust formed at the superfast spreading East Pacific Rise (220 mm/y full spreading rate) (Wilson, 1996) on the Cocos plate in the eastern equatorial Pacific. This site was initially chosen to exploit the inverse relationship between spreading rate and depth to axial low-velocity zones, thought to be magma chambers now frozen as gabbros, observed from seismic experiments (Wilson et al., 2006). Hole 1256D penetrated the entire upper oceanic crust and finally drilled into the uppermost gabbro. Site 1256 is a potential location for a complete penetration of the entire ocean crust in the frame of a "mission," a new operational tool of IODP ("Mission Moho") (Christie et al., 2006; Dick et al., 2006). Initial results of drilling at Site 1256, including detailed site maps and information about the geological setting, can be found in the "Expedition 309/312 summary" chapter and Teagle, Wilson, Acton, and Vanko (2007). The first individual scientific results con- 
cerning the structure/composition of the extrusive rocks and details of the hydrothermal alteration within the lavas and dikes are now available (Busigny et al., 2005; Laverne et al., 2006). In this study, 56 samples of the overlying basaltic lava flows and sheeted dikes, drilled during Leg 206 and Expedition 309, were investigated by light microscope and electron microprobe analyses.

\section{Methods and materials}

Petrographic data were collected from 56 thin sections covering the depth range from 324 to 1255 meters below seafloor (mbsf) in the drill hole, including the following lithostratigraphic units: the "lava pond," the inflated flows, the sheet and massive flows, the transition zone, and the upper part of the sheeted dike complex (Fig. F1). Concerning petrographic terms and rock names we follow those from the "Site 1256" chapter and Wilson, Teagle, Acton, et al. (2003). Phenocryst abundances were estimated by digital images processed in a similar way to that described in the "Methods" chapter.

Primary magmatic phases in 16 basalts were analyzed (red points, Fig. F1) by using polished thin sections and a Cameca SX 100 electron microprobe equipped with five spectrometers and "Peak sight" software. All measurements were made at $15 \mathrm{kV}$ beam potential and $15 \mathrm{nA}$ beam current, with a focused beam and a count time of $20 \mathrm{~s}$ for each element. For some Fe-Ti oxides showing exsolution lamellae, a broad beam $20 \mu \mathrm{m}$ in diameter was used to obtain the initial unexsoluted composition. Matrix correction was performed according to Pouchou and Pichoir (1991). Limits of detection (in weight percent) are as follows:

$$
\begin{aligned}
& \mathrm{SiO}_{2} \text { in Fe-Ti oxides }=0.05, \\
& \mathrm{TiO}_{2} \text { in pyroxene }=0.03, \\
& \mathrm{Al}_{2} \mathrm{O}_{3} \text { in Fe-Ti oxides }=0.09, \\
& \mathrm{Cr}_{2} \mathrm{O}_{3} \text { in pyroxene }=0.18, \\
& \mathrm{Cr}_{2} \mathrm{O}_{3} \text { in Fe-Ti oxides }=0.19, \\
& \mathrm{MgO} \text { in plagioclase }=0.04, \\
& \mathrm{MgO} \text { in Fe-Ti oxides }=0.05, \\
& \mathrm{CaO} \text { in Fe-Ti oxides }=0.05, \\
& \mathrm{Na}_{2} \mathrm{O} \text { in pyroxene }=0.08, \\
& \mathrm{Na}_{2} \mathrm{O} \text { in Fe-Ti oxides }=0.13, \\
& \mathrm{~K}_{2} \mathrm{O} \text { in silicates }=0.08, \text { and } \\
& \mathrm{K}_{2} \mathrm{O} \text { in Fe-Ti oxides }=0.13 .
\end{aligned}
$$

For establishing relationships between mineral and bulk compositions, we chose samples for which bulk analyses are available (see the "Site 1256" chapter and Teagle, Wilson, Acton, and Vanko, 2007).

\section{Petrographic results}

Petrographic results are presented in Table T1. As phenocrysts, only clinopyroxene and plagioclase were identified. Olivine seems to have been completely altered to secondary minerals, as we only identified possible olivine pseudomorphoses. In spite of careful investigation by both optical microscope and backscattered electron (BSE) imaging combined with energy-dispersive X-ray (EDX) analyses, we were not able to find fresh olivine in our samples. The primary groundmass is composed of plagioclase, clinopyroxene, and Fe-Ti oxides, and anhedral clinopyroxene fills the interstices between the mostly euhedral plagioclase laths.

\section{Microanalytical results}

Compositions of the analyzed primary magmatic phases (clinopyroxene, pigeonite, plagioclase, and Fe-Ti oxides) occurring in the investigated basalts are shown in Table T2.

\section{Pyroxenes}

Analyzed clinopyroxenes show strong zonation with decreasing Mg\# toward the rims. Their core compositions vary between $\mathrm{En}_{45} \mathrm{Fs}_{19} \mathrm{Wo}_{36}$ and $\mathrm{En}_{58} \mathrm{Fs}_{11} \mathrm{Wo}_{31}$ with $\mathrm{Mg} \#$ varying from 70.4 to 84.1 . The rims show marked iron enrichment (Fig. F2), with $\mathrm{Mg \#}$ as low as 42.0 , corresponding to a $\mathrm{FeO}^{\text {tot }}$ concentration of $24.6 \mathrm{wt} \%$. Phenocrysts are slightly more primitive in composition compared to groundmass crystals, as reflected by the slightly higher Mg\# (Fig. F3A). As expected, the $\mathrm{Mg \#}$ of the clinopyroxenes correlates with the Mg\# of the corresponding bulk rock (Fig. F3A).

\section{Relics of pigeonite as cores in augite}

Pigeonite has been reported as a groundmass mineral occurring as discrete crystal and thin prismatic cores sandwiched by augite in some lavas (e.g., Crispini et al., 2006; Umino, 2007). Our analyses show that these pigeonites have $\mathrm{CaO}$ contents ranging from 4.4 to $5.2 \mathrm{wt} \%$. $\mathrm{Mg} \#$ of pigeonites in augite ranges from 67.3 to 82.2 and are generally slightly higher than those of the host. Two textural types of pigeonite were observed in the samples:

1. Type I pigeonites (Figs. F4, F5A) correspond to those described in Crispini et al. (2006) and Umino (2007) and can be easily observed under the light microscope as elongated prismatic crystals embedded in augite with a sharp contact between the two. Both pigeonite and augite show 
strong variations in $\mathrm{Mg \#}$. This textural type occurs in the fine-grained samples of Unit 1256D1 , which has been interpreted as a ponded lava flow, as well as in one fine-grained sample (3091256D-118R-1, 43-48 cm) of the flow-dike transition.

2. Type II pigeonites (Figs. F5B, F5C) show different textural features and were first observed in cryptocrystalline and microcrystalline samples of the sheet flows via BSE images. In contrast to Type I pigeonites, they appear as strongly disrupted, often cloudy diffuse patches in intensely zoned groundmass clinopyroxenes, meaning that single pyroxenes bear one or multiple diffuse cores of pigeonitic composition with a more diffuse contact between each other. Because of the strong zonation of the host clinopyroxene, Type II pigeonite is difficult to detect with the light microscope.

The observation of pigeonite in Hole $1256 \mathrm{D}$ is significant. Although low-Ca pyroxene is present in many gabbroic rocks from the plutonic crust of fast-spreading ridges (e.g., Hole 1256D; see the "Site 1256" chapter and Gillis, Mével, Allan, et al., 1993), it is normally not observed as phenocrysts in the corresponding erupted lavas ("the orthopyroxene paradoxon"). Here, low-Ca pyroxene was detected, but only as relics with the composition of pigeonite and not of orthopyroxene, which is the typical low-Ca pyroxene occurring in gabbros. Future studies will shed light on this phenomenon.

\section{Sector zoning}

Figure F6 presents a BSE image of clinopyroxene showing two domains that can be distinguished by a small difference in their gray levels. The brighter zone is, relative to the darker zone, enriched in $\mathrm{Al}$, $\mathrm{Ti}, \mathrm{Cr}$, and $\mathrm{Ca}$ and impoverished in Fe and $\mathrm{Mg}$ (Fig. F6). Such zoning is hard to detect during routine microprobe analysis and is probably the reason for the relatively large standard deviations related to some averages (Table T2).

\section{Plagioclase}

In general, groundmass plagioclase shows marked zoning, with a significant decrease in An content toward the rims. Although the An content of the cores varies between 55.5 and $69.3 \mathrm{~mol} \%$, the rims are much lower in An, ranging from 34.7 to $67.1 \mathrm{~mol} \%$. The standard deviation for the analyses are large, and some cores show exceptionally high An contents up to $>80 \mathrm{~mol} \%$. Figure F7 shows some concentration profiles for An to illustrate zoning trends ob- served in groundmass plagioclases. An and $\mathrm{FeO}^{\text {tot }}$ content in the plagioclase are negatively correlated, suggesting the bulk rock evolution trend to more iron-rich composition with increasing differentiation (Fig. F8). This is confirmed by a diagram of An content versus bulk $\mathrm{Mg \#}$ of the host rocks that shows a positive correlation (Fig. F3B).

Analyzed phenocrysts are significantly enriched in An content relative to the groundmass plagioclase, reaching values up to $83.9 \mathrm{~mol} \%$ (Figs. F3B, F8). This enrichment implies that these phenocrysts are out of equilibrium with the groundmass, representing obvious crystallization products of more primitive magmas, although rims have lower An (<70.9 mol\%). Some plagioclase phenocrysts are completely altered to albite, probably because of secondary hydrothermal alteration.

\section{Fe-Ti oxides}

Coexisting magnetite and ilmenite were found in only one sample of the lava pond (Fig. F9). For this sample, equilibration temperature and oxygen fugacity was calculated using the "QUILF" software (Andersen, 1993). Equilibrium temperature was estimated to be $784^{\circ} \pm 95^{\circ} \mathrm{C}$, reflecting obvious reequilibration during cooling. Calculated oxygen fugacity corresponds to $\triangle \mathrm{FMQ}$ of -0.4 (0.4 log units below the oxygen fugacity of the fayalite-magnetitequartz buffer), which is within the range of oxygen fugacities observed in fresh mid-ocean-ridge basalt (MORB) glasses (Bezos and Humler, 2005).

All other samples contain only titanomagnetite. In the diagram for Fe-Ti oxides (Fig. F10), they plot on the ulvospinel-rich side of the ulvospinel-magnetite solid solution.

\section{Mineral downhole variations}

We observed slightly lower iron content and a slightly higher An in plagioclase, along with a higher clinopyroxene $\mathrm{Mg} \#$ in basalt, in the sheeted dikes, compared to the immediately overlying flows (Fig. F11), but no overall trend directly correlated to depth.

\section{Differentiation trend}

Kvassnes et al. (2004) estimated typical differentiation trends for MORB by plotting Mg\# of clinopyroxene versus An contents of plagioclase in gabbros from different tectonic settings ("dry" and "wet" fractionation). As expected, our data reveal a trend typical for "dry" fractionation (Fig. F12). Including the corresponding mineral data from the two gabbro 
screens in the future will help clarify the genetic relationship between gabbros and basalts from the drilled section.

\section{Acknowledgments}

This study used samples provided by the Integrated Ocean Drilling Program. The project is funded by the Deutsche Forschungsgemeinschaft (DFG).

\section{References}

Andersen, D.J., Lindsley, D.H., and Davidson, P.M., 1993. QUILF: a Pascal program to assess equilibria among FeMg-Mn-Ti oxides, pyroxenes, olivine, and quartz. Comp. Geosci., 19(9):1333-1350. doi:10.1016/00983004(93)90033-2

Bezos, A., and Humler, E., 2005. The $\mathrm{Fe}^{3+} / \Sigma \mathrm{Fe}$ ratios of MORB glasses and their implications for mantle melting. Geochim. Cosmochim. Acta, 69(3):711-725. doi:10.1016/j.gca.2004.07.026

Busigny, V., Laverne, C., and Bonifacie, M., 2005. Nitrogen content and isotopic composition of oceanic crust at a superfast spreading ridge: a profile in altered basalts from ODP Site 1256, Leg 206. Geochem., Geophys., Geosyst., 6(12):Q02O01. doi:10.1029/2005GC001020

Christie, D.M., Ildefonse, B., Abe, N., Arai, S., Bach, W., Blackman, D.K., Duncan, R., Hooft, E., Humphris, S.E., and Miller, D.J., 2006. Mission Moho: formation of oceanic lithosphere. Eos, Trans. Am. Geophys. Union, 87(48):539. doi:10.1029/2006EO480005

Crispini, L., Tartarotti, P., and Umino, S., 2006. Microstructural features of a subaqueous lava from basaltic crust off the East Pacific Rise (ODP Site 1256, Cocos plate). Ofioliti, 31:117-127.

Dick, H.J.B., Natland, J.H., and Ildefonse, B., 2006. Past and future impact of deep drilling in the oceanic crust and mantle. Oceanography, 19(4):74-82.

Gillis, K., Mével, C, Allan, J., et al., 1993. Proc. ODP, Init. Repts., 147: College Station, TX (Ocean Drilling Program). doi:10.2973/odp.proc.ir.147.1993

Kvassnes, A.J.S., Strand, A.H., Moen-Eikeland, H., and Pedersen, R.B., 2004. The Lyngen Gabbro: the lower crust of an Ordovician incipient arc. Contrib. Mineral. Petrol., 148(3):358-379. doi:10.1007/s00410-004-0609-8

Laverne, C., Grauby, O., Alt, J.C., and Bohn, M., 2006. Hydroschorlomite in altered basalts from Hole 1256D,
ODP Leg 206: the transition from low-temperature to hydrothermal alteration. Geochem., Geophys., Geosyst., 7(10):Q10003. doi:10.1029/2005GC001180

Morimoto, N., Fabries, J., Ferguson, A.K., Ginzburg, I.V., Ross, M., Seifert, F.A., Zussman, J., Aoki, K., and Gottardi, G., 1988. Nomenclature of pyroxenes. Am. Mineral., 73(9-10):1123-1133.

Pouchou, J.-L., and Pichoir, F., 1991. Quantitative analysis of homogeneous of stratified micro-volumes applying the model "PAP." In: Heinrich, K.F.J., and Newberry, D.E. (Eds.), Electron Probe Quantitation: New York (Plenum), 31-75.

Teagle, D.A.H., Wilson, D.S., Acton, G.D., and Vanko, D.A. (Eds.), 2007. Proc. ODP., Sci. Results, 206: College Station, TX (Ocean Drilling Program). doi:10.2973/ odp.proc.sr.206.2007

Umino, S., 2007. Data report: textural variation of Units 1256C-18 and 1256D-1 lava pond, with special reference to recrystallization of the base of Unit 1256C-18. In Teagle, D.A.H., Wilson, D.S., Acton, G.D., and Vanko, D.A. (Eds.), Proc. ODP, Sci. Results, 206: College Station, TX (Ocean Drilling Program), 1-32. doi:10.2973/ odp.proc.sr.206.007.2007

Wilson, D.S., 1996. Fastest known spreading on the Miocene Cocos-Pacific plate boundary. Geophys. Res. Lett., 23(21):3003-3006. doi:10.1029/96GL02893

Wilson, D.S., Teagle, D.A.H., Acton, G.D., et al., 2003. Proc. ODP, Init. Repts., 206: College Station, TX (Ocean Drilling Program). doi:10.2973/odp.proc.ir.206.2003

Wilson, D.S., Teagle, D.A.H., Alt, J.C., Banerjee, N.R., Umino, S., Miyashita, S., Acton, G.D., Anma, R., Barr, S.R., Belghoul, A., Carlut, J., Christie, D.M., Coggon, R.M., Cooper, K.M., Cordier, C., Crispini, L., Durand, S.R., Einaudi, F., Galli, L., Gao, Y., Geldmacher, J., Gilbert, L.A., Hayman, N.W., Herrero-Bervera, E., Hirano, N., Holter, S., Ingle, S., Jiang, S., Kalberkamp, U. Kerneklian, M., Koepke, J., Laverne, C., Vasquez, H.L.L., Maclennan, J., Morgan, S., Neo, N., Nichols, H.J., Park, S.-H., Reichow, M.K., Sakuyama, T., Sano, T., Sandwell, R., Scheibner, B., Smith-Duque, C.E., Swift, S.A., Tartarotti, P., Tikku, A.A., Tominaga, M., Veloso, E.A., Yamasaki, T., Yamazaki, S., and Ziegler, C., 2006. Drilling to gabbro in intact ocean crust. Science, 312(5776):1016-1020. doi:10.1126/science.1126090

Initial receipt: 2 January 2008

Acceptance: 23 June 2008

Publication: 6 October 2008

MS 309312-201 
Figure F1. Simplified lithostratigraphy according to Teagle, Wilson, Acton, and Vanko (2006), including locations of investigated (white) and analyzed (red) samples.

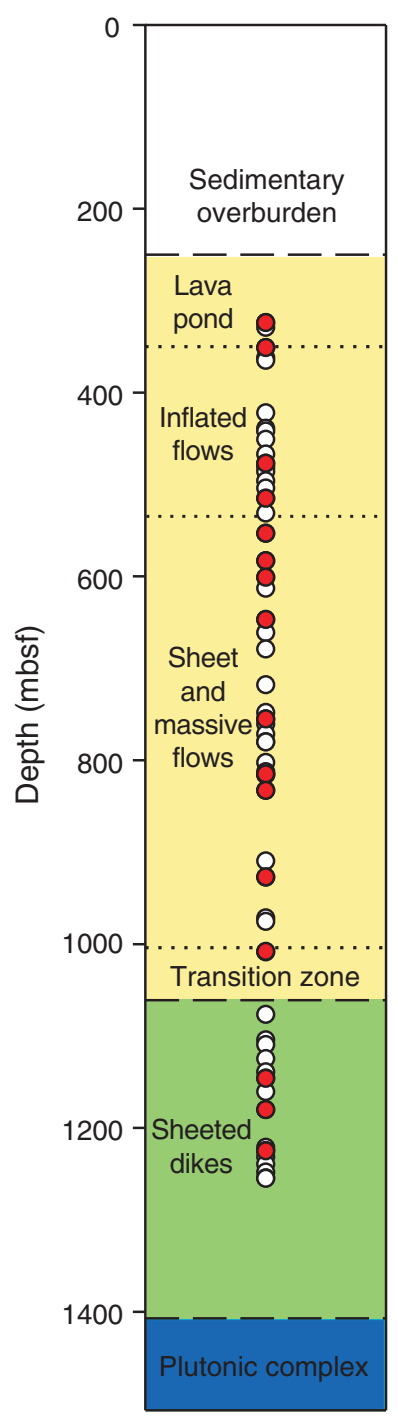


Figure F2. Average compositions of pyroxenes. Quadrilateral after Morimoto et al. (1988). Di = diopside, Hd = hedenbergite, $\mathrm{En}=$ enstatite, $\mathrm{Fs}=$ ferrosilite.

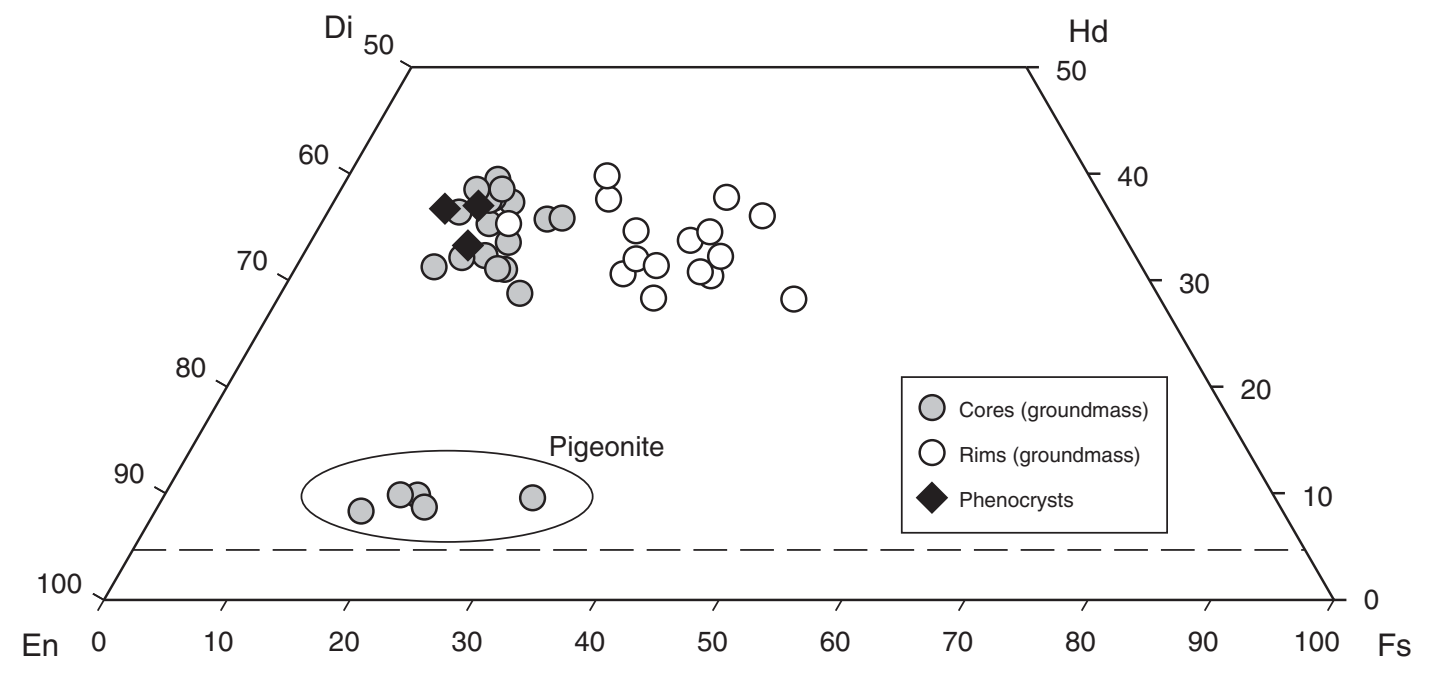


Figure F3. Mg\# of clinopyroxene (only core compositions) vs. Mg\# of corresponding bulk rock.
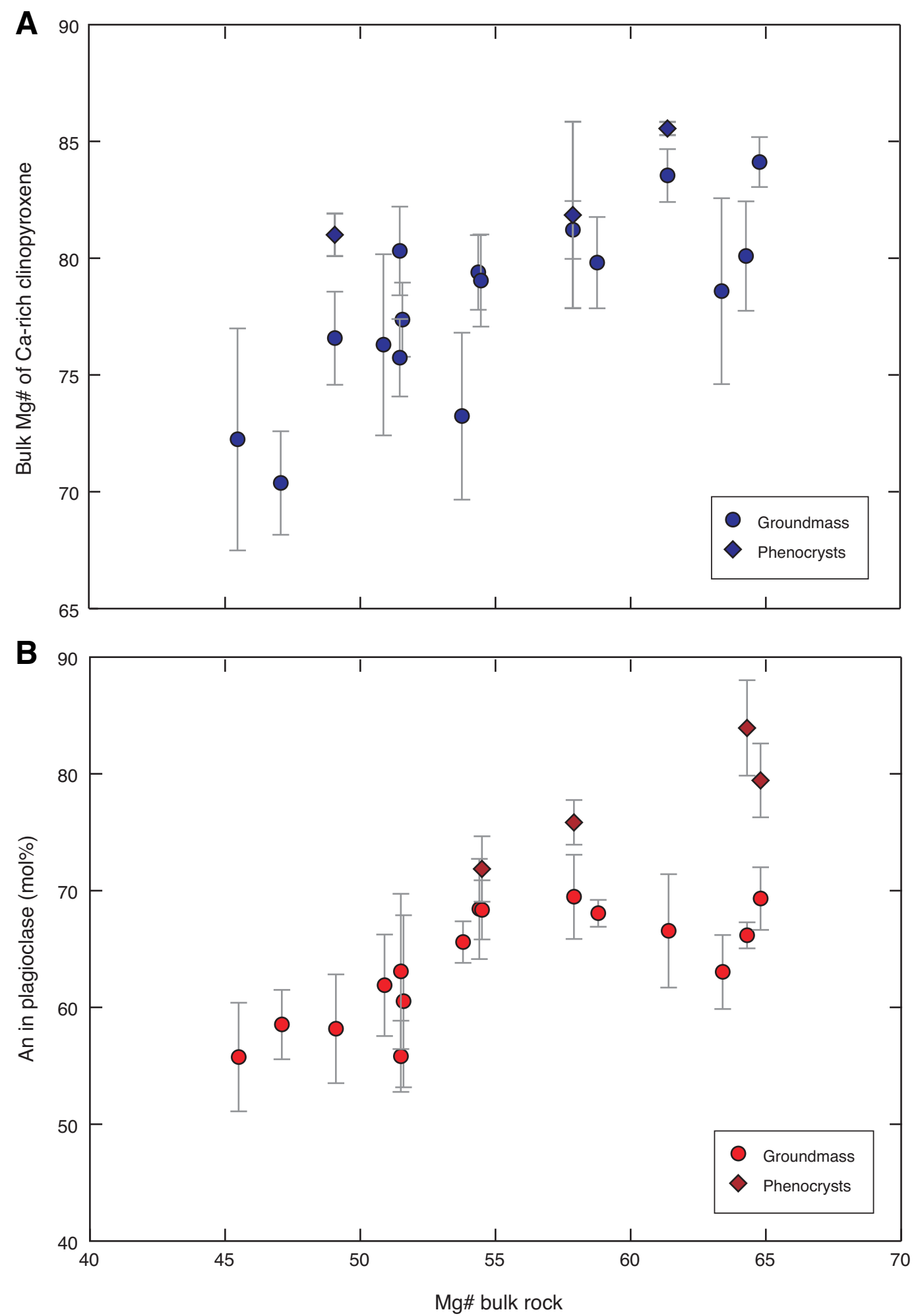
Figure F4. Photomicrograph of groundmass clinopyroxene (cpx) bearing core of Type I relict pigeonite (pgt) (cross-polarized light) (see text for details).

Sample 206-1256D-10R-2, 28-30 cm

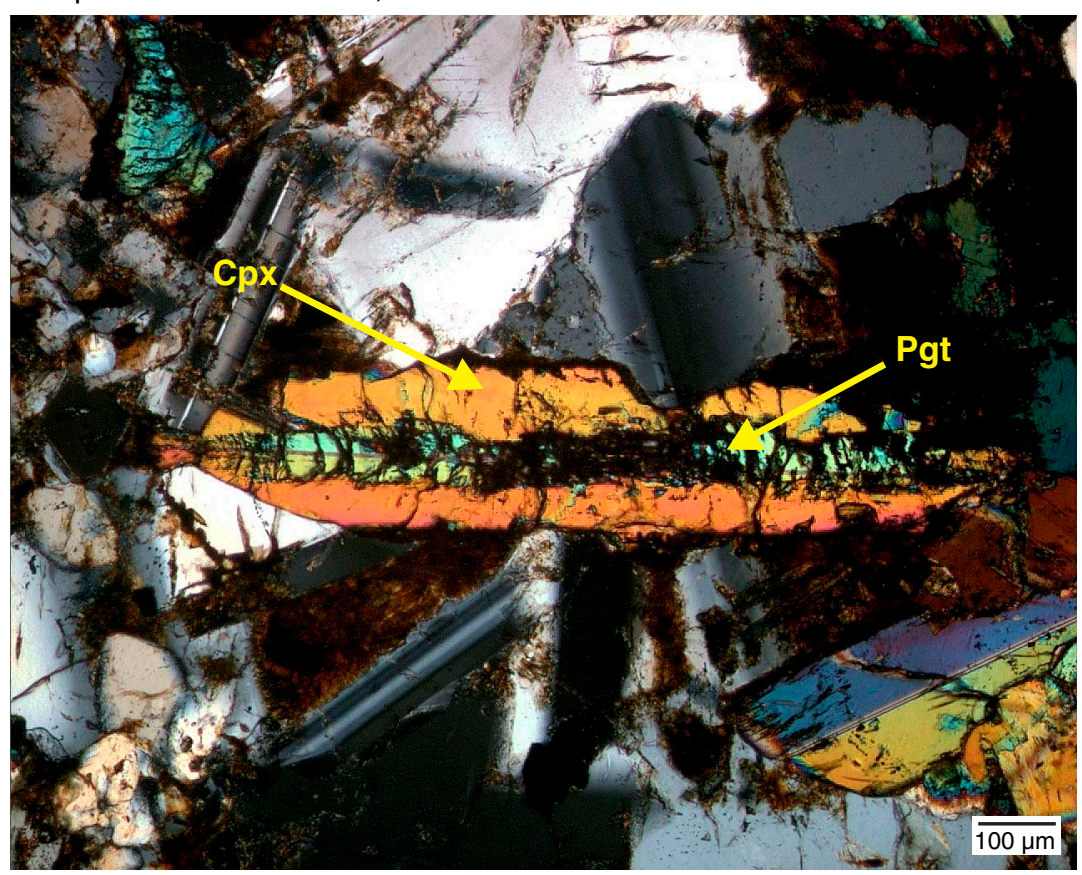


Figure F5. BSE images of clinopyroxene (cpx) with cores of relict pigeonite (pgt). A. Type I pigeonite. B, C. Type II pigeonite and related electron microprobe profiles (see text for details). Dashed arrows indicate location of profile. $\mathrm{pl}=$ plagioclase.

A Sample 206-1256D-9R-2, 7-10 cm

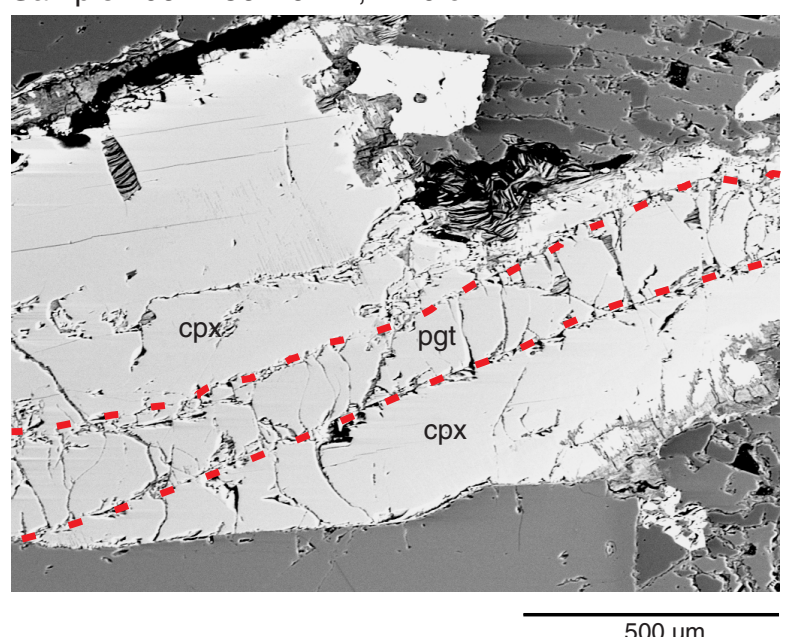

B Sample 206-1256D-39R-2, 9-10 cm

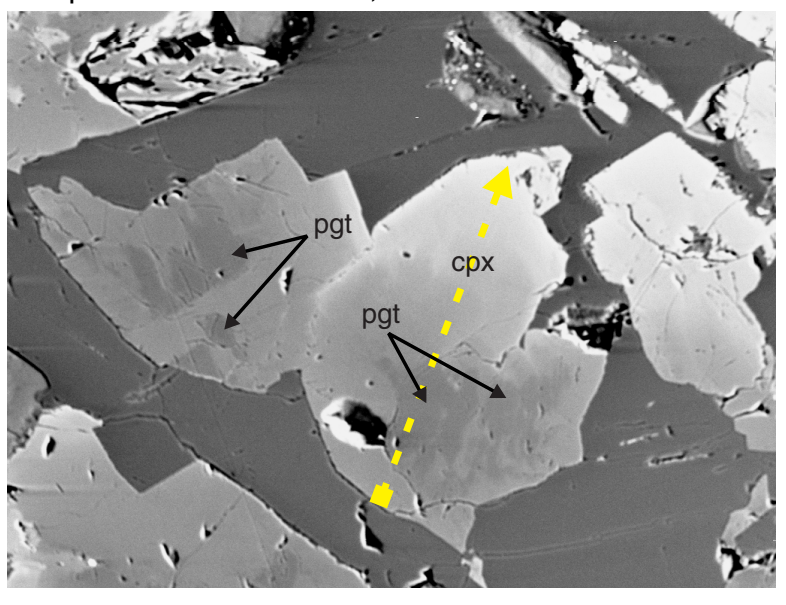

C Sample 206-1256D-52R-1, 5-7 cm

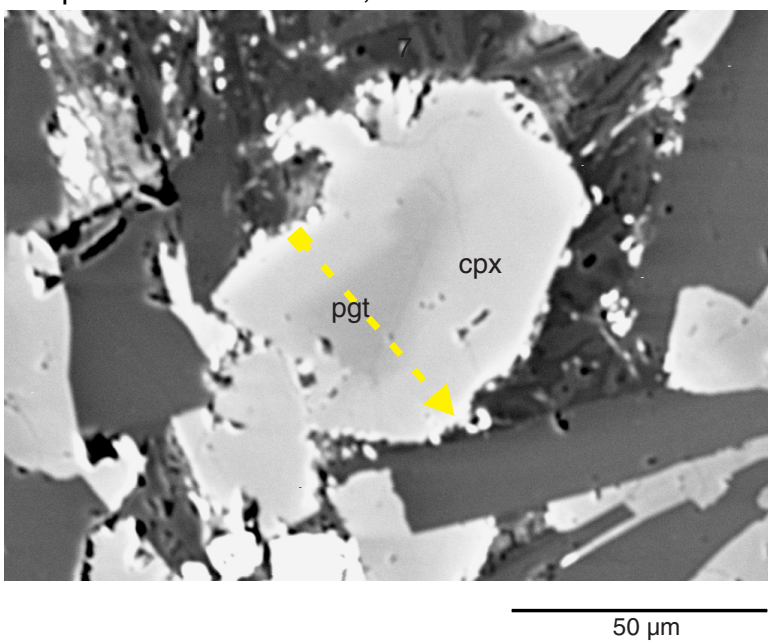

Sample 206-1256D-9R-2, 7-10 cm
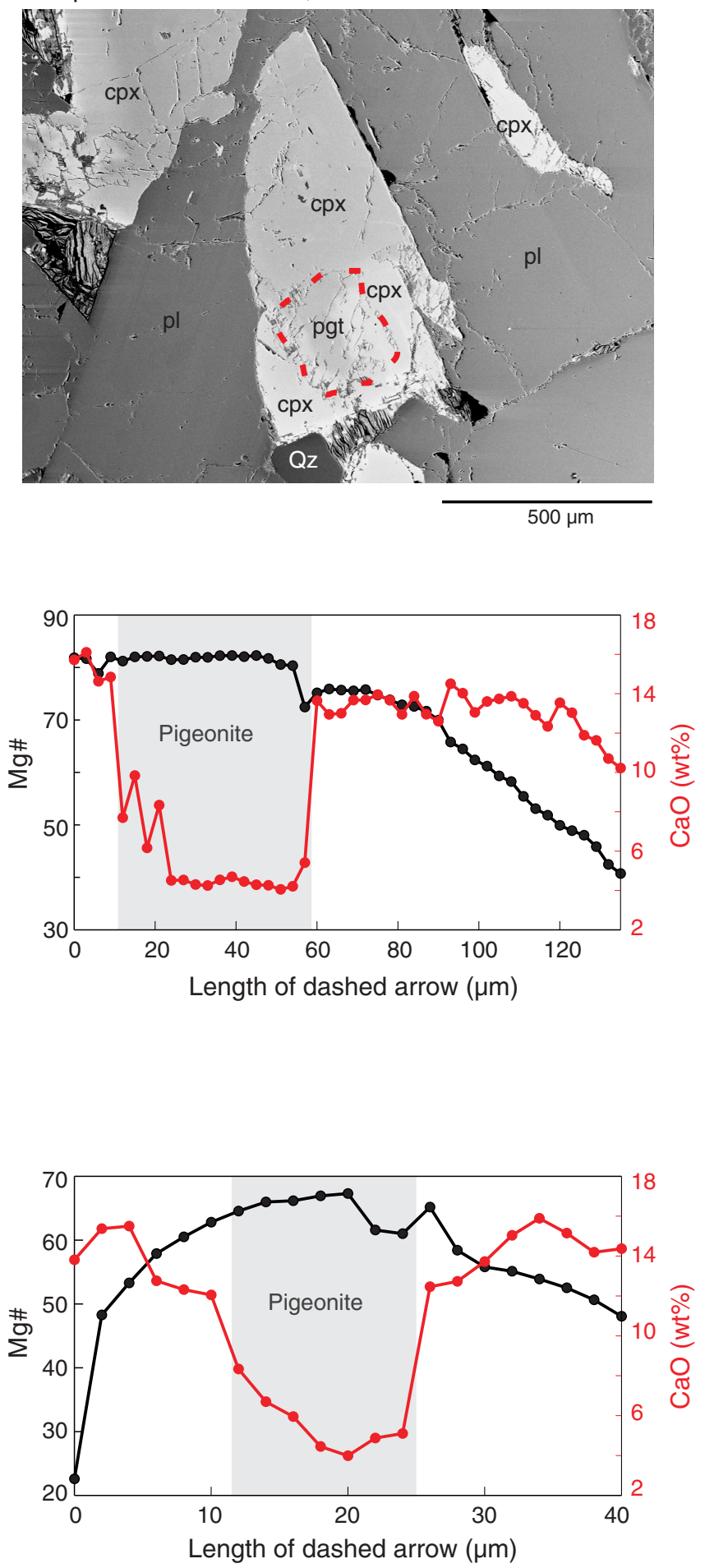
Figure F6. BSE image of clinopyroxene crystal with marked sector zoning. Brighter zone in middle of crystal is relatively enriched in $\mathrm{Al}$, $\mathrm{Ti}$, $\mathrm{Cr}$, and $\mathrm{Ca}$, whereas $\mathrm{Fe}$ and $\mathrm{Mg}$ are depleted. Dashed arrow indicates location of corresponding microprobe profile through this grain (Fig. F7).

Sample 206-1256D-57R-2, 24-26 cm

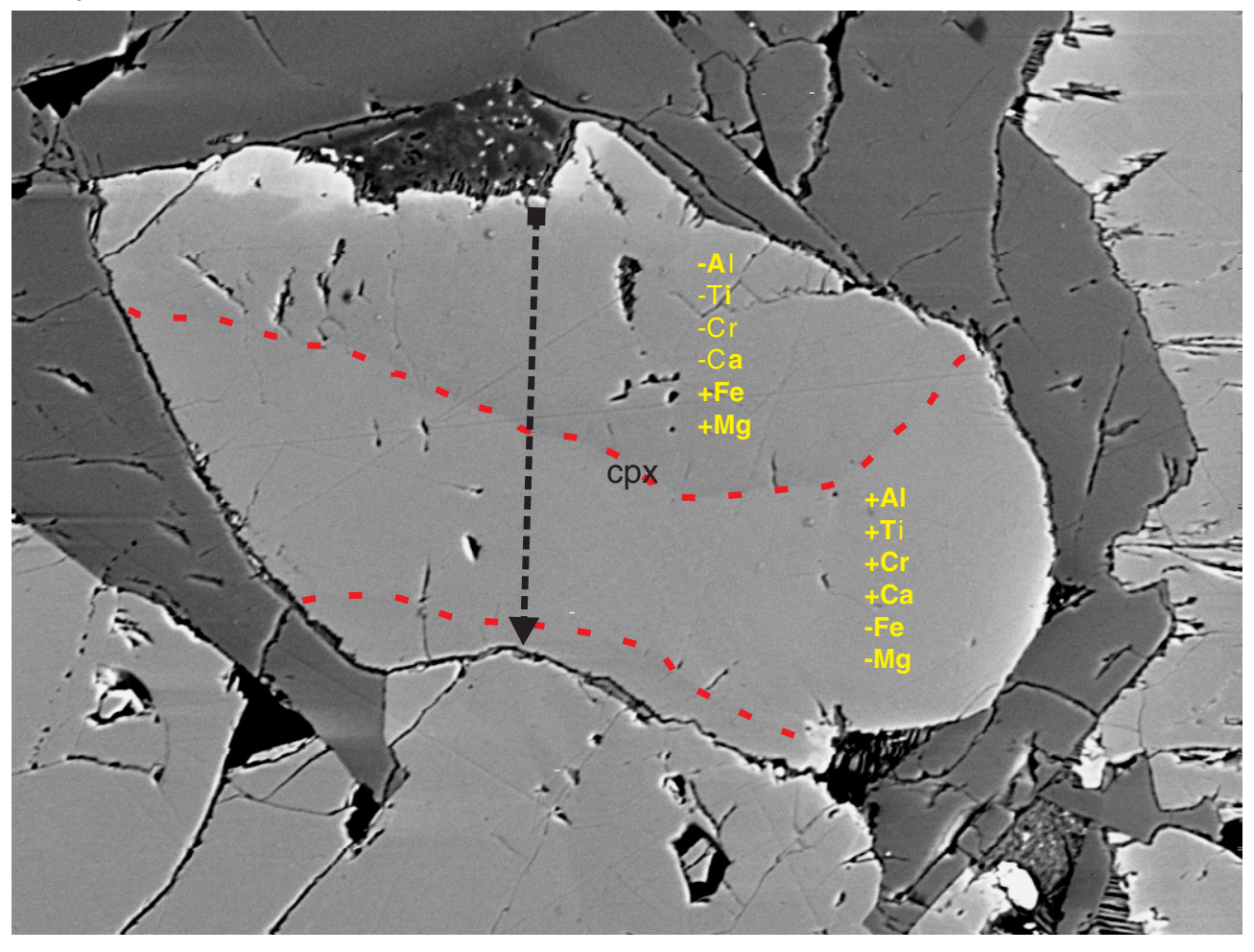

$100 \mu \mathrm{m}$ 
Figure F7. Microprobe profile for elements through clinopyroxene with marked sector zoning shown in Figure F6.

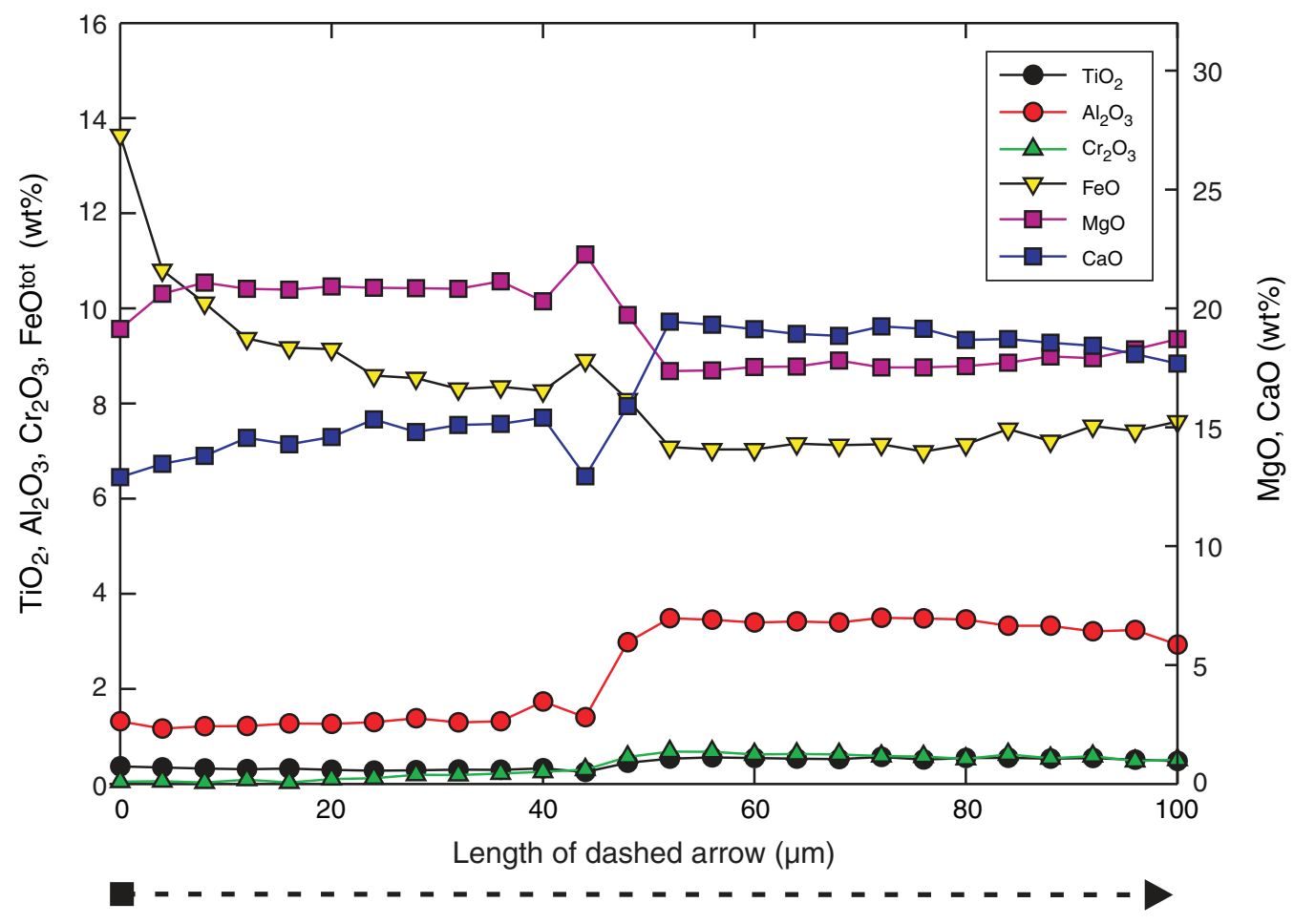


Figure F8. BSE images of groundmass plagioclase (pl) and related electron microprobe profiles for An content. Dashed arrows indicate location of profile.

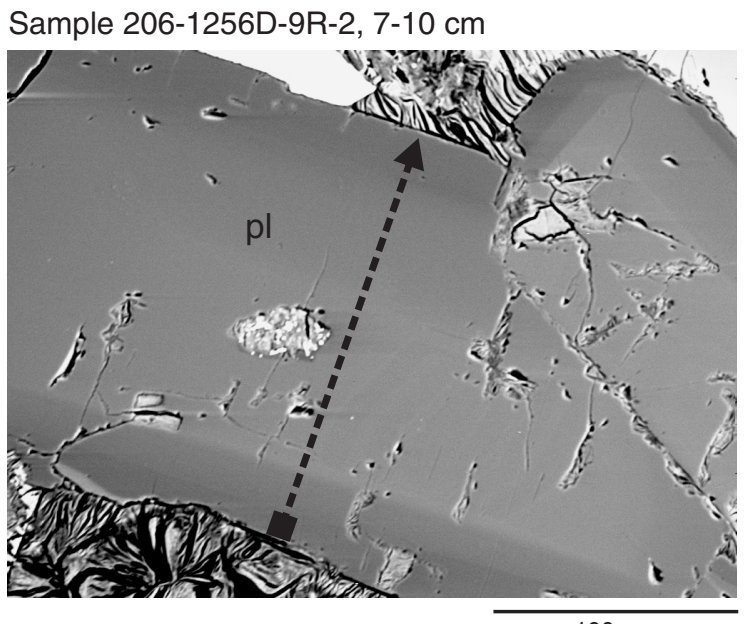

Sample 206-1256D-49R-2, 38-40 cm

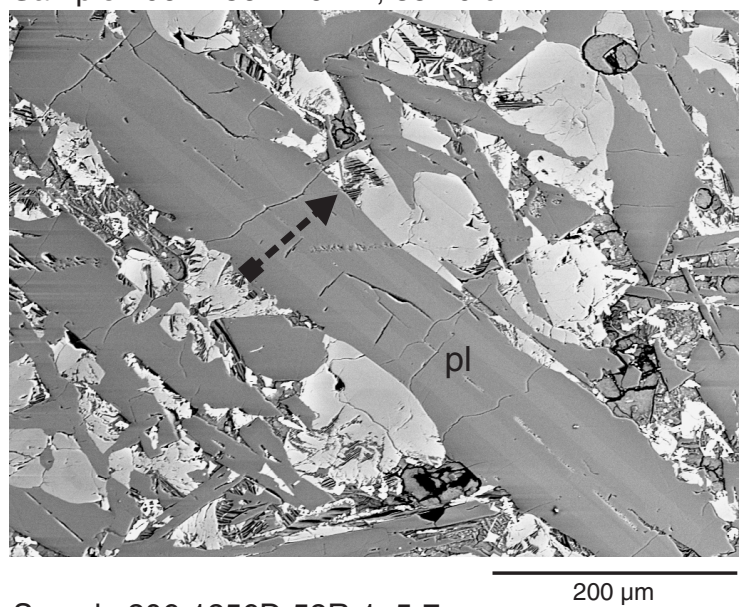

Sample 206-1256D-52R-1, 5-7 cm

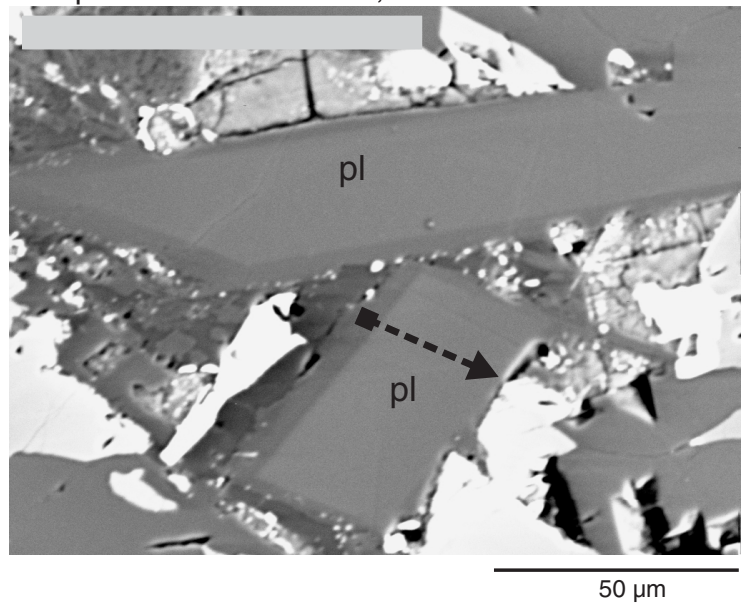

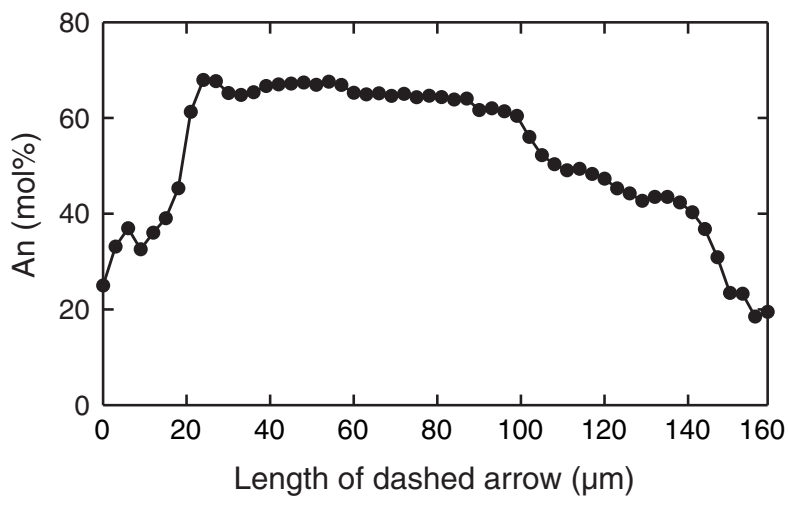
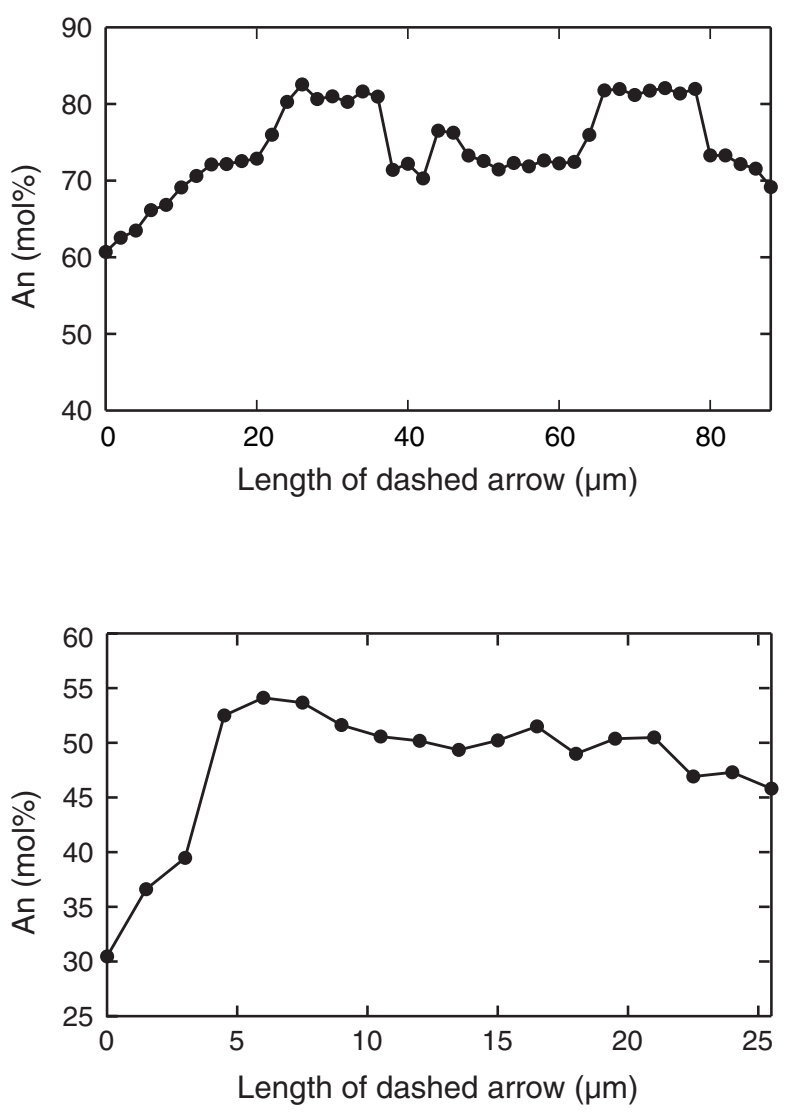
Figure F9. FeO $^{\text {tot }}$ in plagioclase vs. An content (only core compositions).

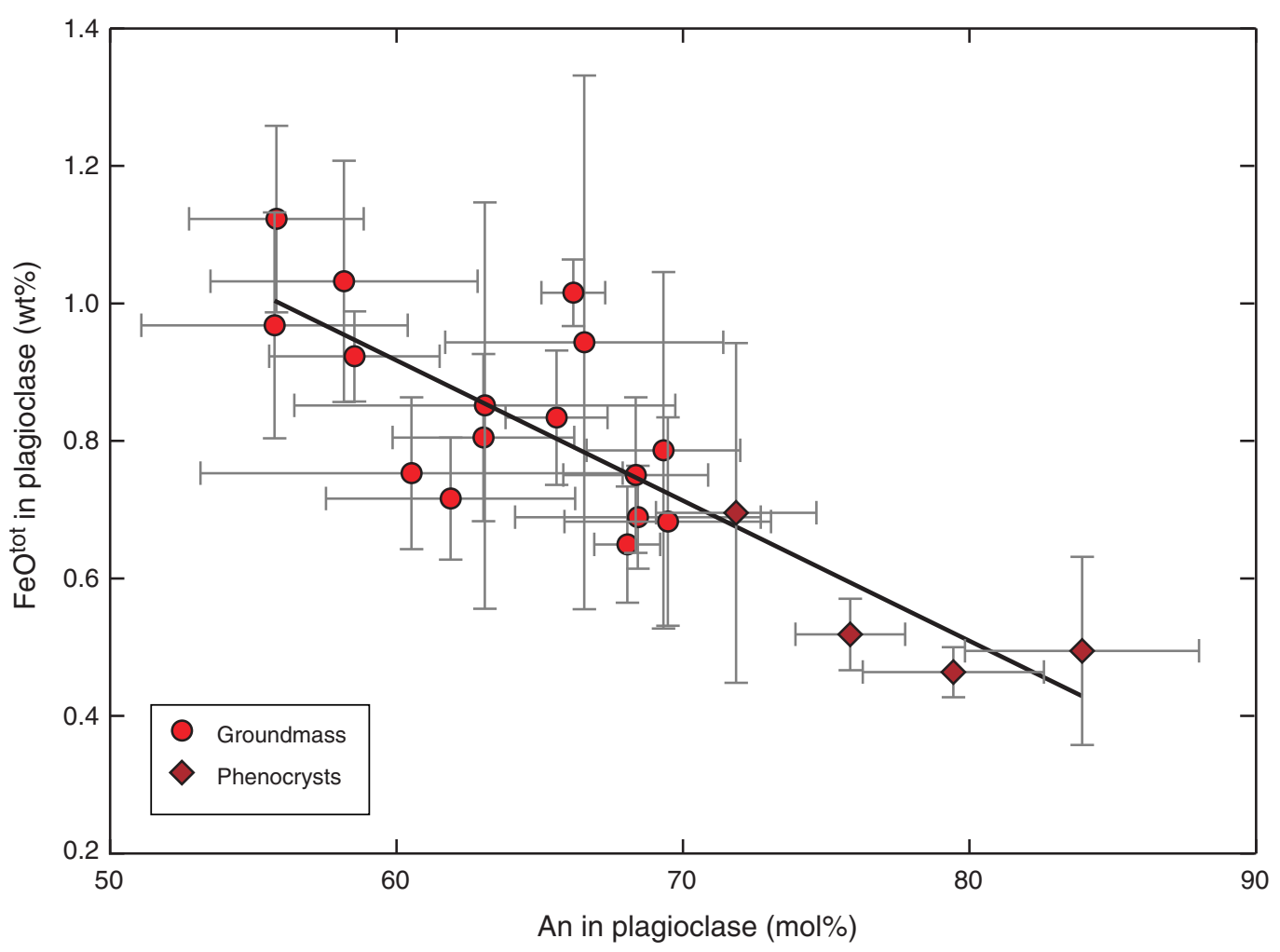


Figure F10. BSE image of oxide cluster with coexisting magnetite (mt) and ilmenite (il).

Sample 206-1256D-9R-2, 7-10 cm

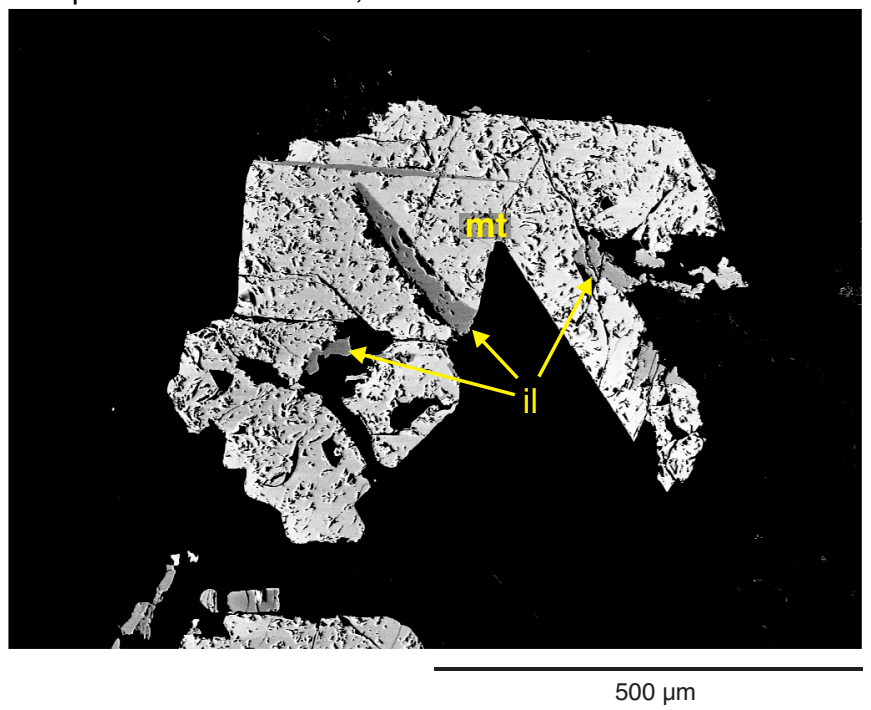


Figure F11. Plot of analyzed magnetites and ilmenites in $\mathrm{FeO}-\mathrm{Fe}_{2} \mathrm{O}_{3}-\mathrm{TiO}_{2}$-ternary diagram.

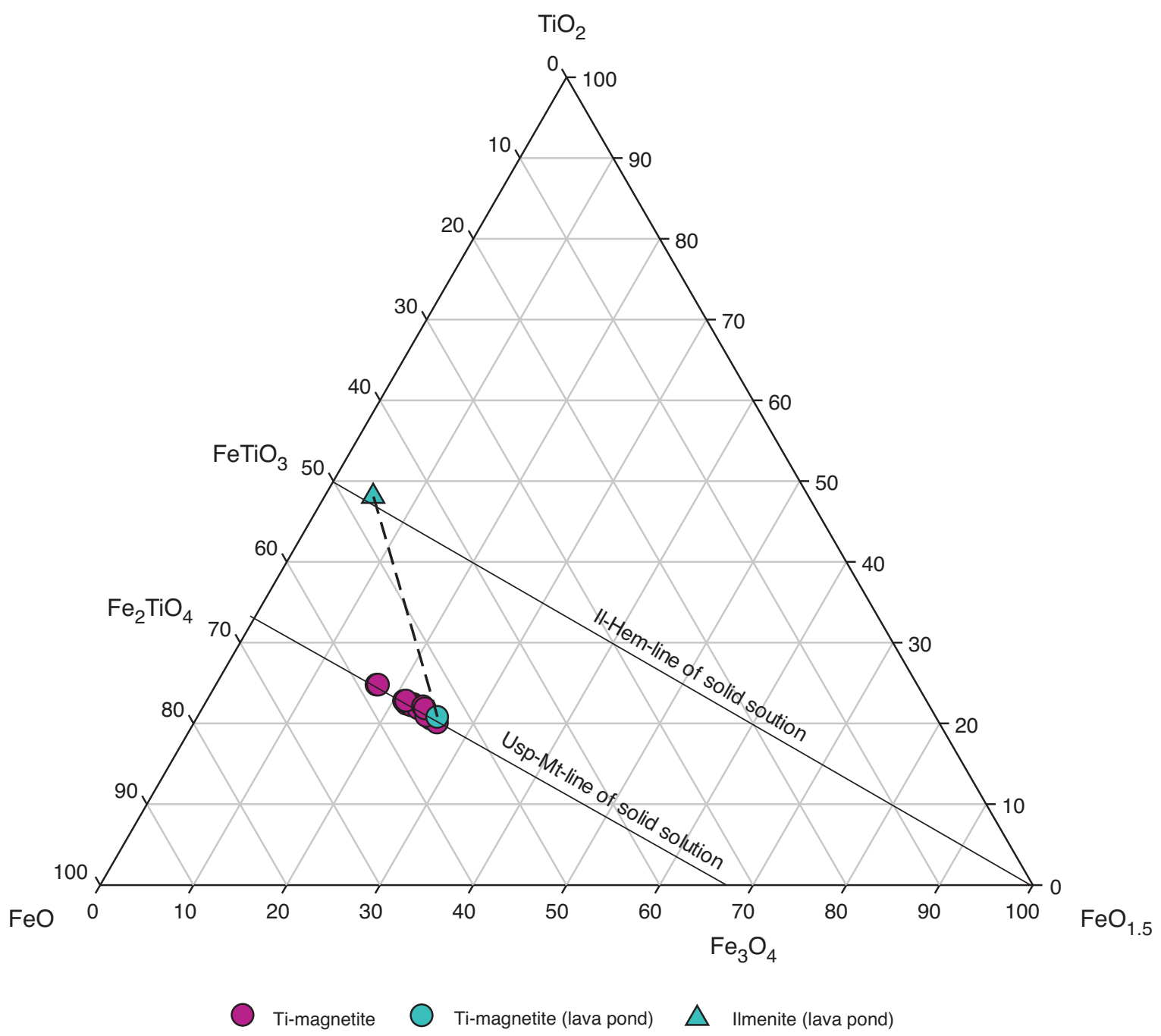


Figure F12. Downhole evolution of An content and $\mathrm{FeO}^{\text {tot }}$ content of groundmass plagioclase and $\mathrm{Mg \#}$ of groundmass clinopyroxene.

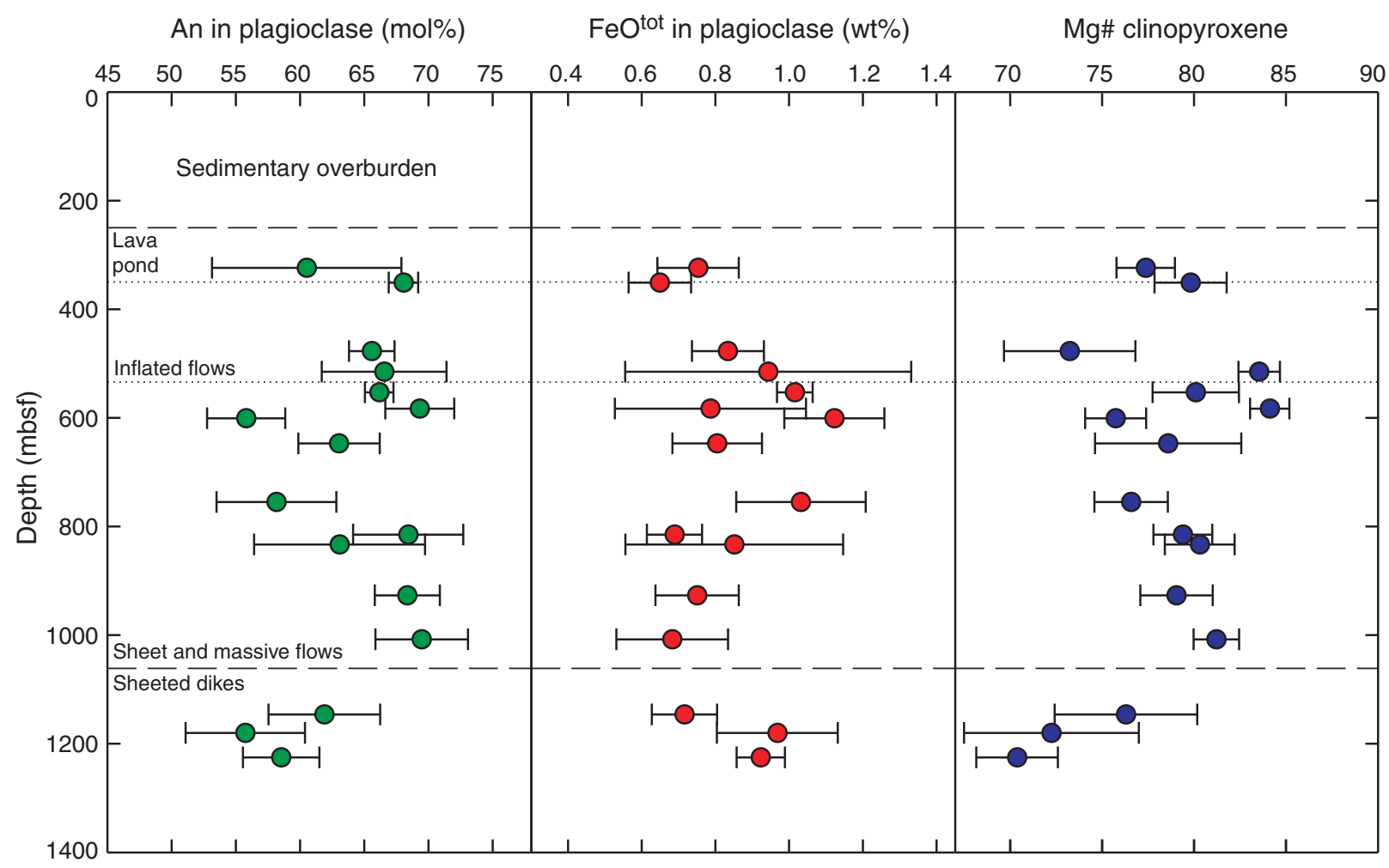


Figure F13. Mg\# of clinopyroxene vs. An content of plagioclase plotted against general fractionation trends (redrawn after Kvassnes et al., 2004).

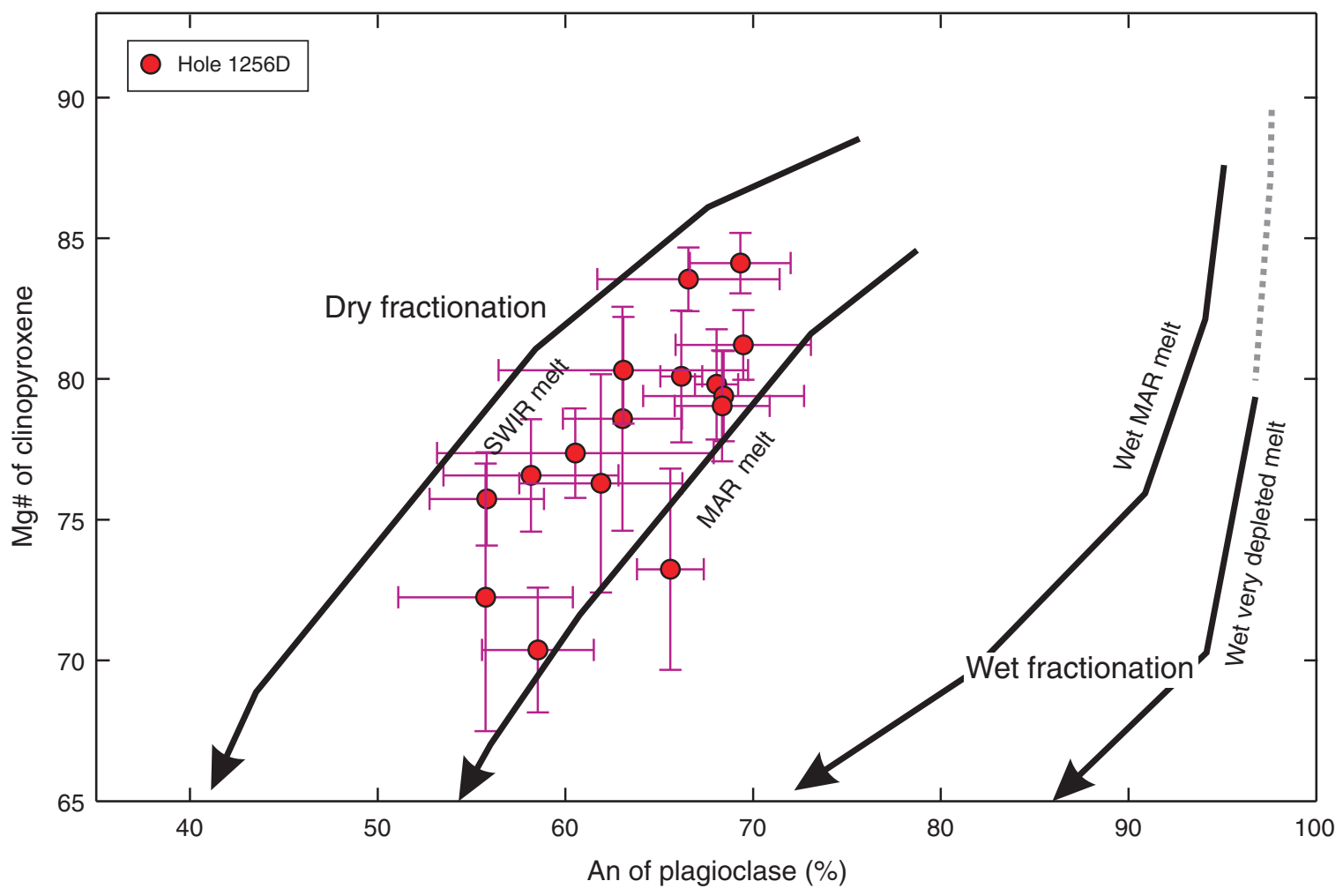


Table T1. Textural features of samples, Hole 1256D. (See table notes.)

\begin{tabular}{|c|c|c|c|c|c|c|c|c|c|}
\hline \multirow{2}{*}{$\begin{array}{l}\text { Core, section, } \\
\text { interval }(\mathrm{cm})\end{array}$} & \multirow{2}{*}{$\begin{array}{l}\text { Depth CSF } \\
\quad(\mathrm{m})\end{array}$} & \multirow{2}{*}{$\begin{array}{c}\text { Mode of } \\
\text { emplacement }\end{array}$} & \multirow{2}{*}{$\begin{array}{l}\text { Phenocryst } \\
\text { texture }\end{array}$} & \multirow[b]{2}{*}{ Size } & \multirow[b]{2}{*}{ Crystallinity } & \multirow[b]{2}{*}{ Texture type } & \multicolumn{3}{|c|}{ Phenocrysts (\%) } \\
\hline & & & & & & & $\mathrm{cpx}$ & $\mathrm{pl}$ & Total \\
\hline \multicolumn{10}{|l|}{ 206-1256D- } \\
\hline 9R-2, 7-10 & 324 & Ponded lava flow & Aphyric & Fine grained & Hypocrystalline & Intersertal & 0.0 & 0.0 & 0.0 \\
\hline $10 \mathrm{R}-2,28-30$ & 329 & Ponded lava flow & Aphyric & Fine grained & Hypocrystalline & Intersertal & 0.0 & 0.0 & 0.0 \\
\hline 13R-1, 109-111 & 351 & Massive or sheet flow & Aphyric & Microcrystalline & Hypocrystalline & Intersertal & 0.0 & 0.5 & 0.5 \\
\hline $14 \mathrm{R}-2,127-130$ & 362 & Massive or sheet flow & Sparsely phyric & Cryptocrystalline & Hypocrystalline & Variolitic & 0.0 & 1.1 & 1.1 \\
\hline 15R-1, 69-71 & 365 & Massive or sheet flow & Aphyric & Cryptocrystalline & Hypocrystalline & Intersertal & 0.0 & 0.0 & 0.0 \\
\hline $24 \mathrm{R}-2,119-122$ & 422 & Massive or sheet flow & Sparsely phyric & Cryptocrystalline & Hypocrystalline & Intersertal-pilotaxitic & 0.5 & 1.3 & 1.8 \\
\hline $26 \mathrm{R}-1,22-24$ & 439 & Massive or sheet flow & Aphyric & Cryptocrystalline & Hypocrystalline & Intersertal & 0.0 & 0.0 & 0.0 \\
\hline 26R-4, 17-19 & 442 & Massive or sheet flow & Aphyric & Microcrystalline & Hypocrystalline & Intersertal & 0.0 & 0.0 & 0.0 \\
\hline $28 \mathrm{R}-1,33-35$ & 451 & Massive or sheet flow & Aphyric & Cryptocrystalline & Hypocrystalline & Variolitic-intersertal & 0.0 & 0.0 & 0.0 \\
\hline $31 \mathrm{R}-1,125-127$ & 467 & Massive or sheet flow & Aphyric & Cryptocrystalline & Hypocrystalline & Variolitic & 0.0 & 0.0 & 0.0 \\
\hline $32 R-6,6-8$ & 477 & Massive or sheet flow & Aphyric & Microcrystalline & Hypocrystalline & Intersertal & 0.0 & 0.0 & 0.0 \\
\hline $33 \mathrm{R}-1,32-35$ & 481 & Massive or sheet flow & Aphyric & Cryptocrystalline & Hypocrystalline & Variolitic-intersertal & 0.0 & 0.0 & 0.0 \\
\hline $34 \mathrm{R}-2,34-37$ & 486 & Massive or sheet flow & Aphyric & Cryptocrystalline & Hypocrystalline & Variolitic-intersertal & 0.0 & 0.0 & 0.0 \\
\hline $36 \mathrm{R}-2,37-39$ & 496 & Massive or sheet flow & Aphyric & Cryptocrystalline & Hypocrystalline & Intersertal & 0.0 & 0.0 & 0.0 \\
\hline $38 \mathrm{R}-1,10-14$ & 504 & Massive or sheet flow & Aphyric & Cryptocrystalline & Hypocrystalline & Intersertal & 0.0 & 0.0 & 0.0 \\
\hline $39 \mathrm{R}-2,9-12$ & 515 & Massive or sheet flow & Aphyric & Microcrystalline & Hypocrystalline & Intersertal & 0.0 & 0.0 & 0.0 \\
\hline $42 \mathrm{R}-2,31-33$ & 531 & Massive or sheet flow & Aphyric & Cryptocrystalline & Hypocrystalline & Variolitic & 0.0 & 0.0 & 0.0 \\
\hline $45 \mathrm{R}-1,92-95$ & 553 & Massive or sheet flow & Aphyric & Cryptocrystalline & Hypocrystalline & Variolitic-seriate & 0.0 & 0.3 & 0.3 \\
\hline $49 \mathrm{R}-2,38-40$ & 583 & Massive or sheet flow & Aphyric & Cryptocrystalline & Hypocrystalline & Variolitic-seriate & 0.0 & 0.7 & 0.7 \\
\hline $52 \mathrm{R}-1,5-7$ & 601 & Massive or sheet flow & Aphyric & Cryptocrystalline & Hypocrystalline & Variolitic & 0.0 & 0.0 & 0.0 \\
\hline $53 R-3,22-24$ & 613 & Massive or sheet flow & Aphyric & Cryptocrystalline & Hypocrystalline & Variolitic & 0.0 & 0.0 & 0.0 \\
\hline $57 R-2,24-26$ & 647 & Massive or sheet flow & Aphyric & Fine grained & Hypocrystalline & Intersertal & 0.0 & 0.0 & 0.0 \\
\hline $59 \mathrm{R}-2,114-115$ & 661 & Massive or sheet flow & Sparsely phyric & Cryptocrystalline & Hypocrystalline & $\begin{array}{l}\text { Variolitic- } \\
\text { glomeroporphyritic }\end{array}$ & 0.8 & 1.0 & 1.8 \\
\hline $61 \mathrm{R}-1,91-96$ & 679 & Massive or sheet flow & Aphyric & Cryptocrystalline & Hypocrystalline & Intersertal & 0.0 & 0.0 & 0.0 \\
\hline $67 R-3,60-65$ & 718 & Massive or sheet flow & Aphyric & Cryptocrystalline & Hypocrystalline & Variolitic & 0.5 & 0.0 & 0.5 \\
\hline $74 \mathrm{R}-1,110-112$ & 748 & Massive or sheet flow & Aphyric & Cryptocrystalline & Hypocrystalline & Variolitic-intersertal & 0.1 & 0.0 & 0.1 \\
\hline \multicolumn{10}{|l|}{ 309-1256D- } \\
\hline $76 \mathrm{R}-2,0-4$ & 755 & Massive or sheet flow & Aphyric & Cryptocrystalline & Hypocrystalline & Variolitic & 0.2 & 0.5 & 0.7 \\
\hline $77 R-2,52-55$ & 761 & Massive or sheet flow & Moderately phyric & Cryptocrystalline & Hypocrystalline & Variolitic & 0.5 & 2.9 & 3.5 \\
\hline $79 \mathrm{R}-2,48-50$ & 772 & Massive or sheet flow & Aphyric & Cryptocrystalline & Hypocrystalline & Intersertal & 0.0 & 0.0 & 0.0 \\
\hline $80 \mathrm{R}-1,55-58$ & 780 & Massive or sheet flow & Aphyric & Cryptocrystalline & Hypocrystalline & Variolitic & 0.0 & 0.0 & 0.0 \\
\hline $80 \mathrm{R}-1,102-103$ & 780 & Massive or sheet flow & Aphyric & Cryptocrystalline & Hypocrystalline & Variolitic & 0.0 & 0.0 & 0.0 \\
\hline $84 \mathrm{R}-1,54-56$ & 802 & Massive or sheet flow & Aphyric & Cryptocrystalline & Hypocrystalline & Variolitic & 0.0 & 0.0 & 0.0 \\
\hline $85 R-1,116-119$ & 813 & Massive o & Aphyric & Microcrystalline & Hypocrystalline & Inters & 0.0 & 0.0 & 0.0 \\
\hline $85 R-3,76-78$ & 815 & Massive or sheet flow & Aphyric & Microcrystalline & Hypocrystalline & Intersertal-subophitic & 0.0 & 0.0 & 0.0 \\
\hline $85 R-4,42-45$ & 816 & Massive or sheet flow & Moderately phyric & $\begin{array}{l}\text { Cryptocrystalline, } \\
\text { fine grained }\end{array}$ & Hypocrystalline & $\begin{array}{c}\text { Variolitic- } \\
\text { glomeroporphyritic }\end{array}$ & 0.1 & 2.1 & 2.3 \\
\hline $87 R-2,63-66$ & 833 & Massive or sheet flow & Moderately phyric & Fine grained & Hypocrystalline & Intersertal-subophitic & 9.1 & 0.0 & 9.1 \\
\hline $99 \mathrm{R}-2,78-82$ & 909 & Massive or sheet flow & Aphyric & Cryptocrystalline & Hypocrystalline & Variolitic & 0.0 & 0.1 & 0.1 \\
\hline 102R-1, 70-72 & 927 & Massive or sheet flow & Aphyric & $\begin{array}{l}\text { Microcrystalline, } \\
\text { fine grained }\end{array}$ & Hypocrystalline & Variolitic-intersertal & 0.0 & 0.3 & 0.3 \\
\hline $110 \mathrm{R}-2,56-60$ & 972 & Massive o & A & Microcrystalline & alline & & 0.0 & 0.0 & 0.0 \\
\hline $111 \mathrm{R}-1,85-87$ & 975 & Massive or sheet flow & Aphyric & Fine grained & Hypocrystalline & Intersertal & 0.2 & 0.3 & 0.5 \\
\hline $118 \mathrm{R}-1,45-48$ & 1008 & Massive or sheet flow & Sparsely phyric & Fine grained & Hypocrystalline & Intersertal & 0.0 & 1.6 & 1.6 \\
\hline $132 \mathrm{R}-1,112-114$ & 1076 & Sheeted dike & Aphyric & Cryptocrystalline & Hypocrystalline & Variolitic & 0.0 & 0.0 & 0.0 \\
\hline $138 \mathrm{R}-1,10-12$ & 1104 & Sheeted dike & Aphyric & Cryptocrystalline & Hypocrystalline & Intersertal & 0.0 & 0.0 & 0.0 \\
\hline $139 \mathrm{R}-1,48-50$ & 1109 & Sheeted dike & Aphyric & Microcrystalline & Hypocrystalline & Variolitic-intersertal & 0.6 & 0.0 & 0.6 \\
\hline $142 \mathrm{R}-1,119-121$ & 1124 & Sheeted dike & Aphyric & Fine grained & Hypocrystalline & $\begin{array}{l}\text { Intergranular- } \\
\text { subophitic }\end{array}$ & 0.0 & 0.0 & 0.0 \\
\hline $145 R-2,51-55$ & 1139 & Sheeted dike & Aphyric & Microcrystalline & Holocrystalline & Intergranular & 0.0 & 0.0 & 0.0 \\
\hline 147R-1, 71-75 & 1146 & Sheeted dike & Aphyric & Fine grained & Holocrystalline & Intergranular & 0.0 & 0.0 & 0.0 \\
\hline 150R-1, 8-10 & 1160 & Sheeted dike & Aphyric & Fine grained & Holocrystalline & Variolitic-intergranular & 0.0 & 0.0 & 0.0 \\
\hline 154R-1, 56-60 & 1180 & Sheeted dike & Aphyric & Microcrystalline & Holocrystalline & Intergranular & 0.0 & 0.0 & 0.0 \\
\hline $163 R-3,59-62$ & 1221 & Sheeted dike & Sparsely phyric & Cryptocrystalline & Holocrystalline & Variolitic & 0.0 & 1.0 & 1.0 \\
\hline $164 \mathrm{R}-2,115-118$ & 1225 & Sheeted dike & Aphyric & $\begin{array}{l}\text { Microcrystalline, } \\
\text { fine grained }\end{array}$ & Holocrystalline & Variolitic-intersertal & 0.0 & 0.0 & 0.0 \\
\hline 165R-3, 102-105 & 1231 & Sheete & Aph & Microcrystalline & $\mathrm{Hol}$ & Variolitic-intersertal & 0.0 & 0.0 & 0.0 \\
\hline $167 R-3,51-53$ & 1239 & Sheeted dike & Aphyric & Cryptocrystalline & Holocrystalline & Variolitic & 0.3 & 0.6 & 0.9 \\
\hline $168 \mathrm{R}-5,35-37$ & 1248 & Sheeted dike & Aphyric & Microcrystalline & Holocrystalline & Variolitic-intersertal & 0.0 & 0.0 & 0.0 \\
\hline 170R-2, 90-93 & 1254 & Sheeted dike & Sparsely phyric & Cryptocrystalline & Holocrystalline & Variolitic & 0.9 & 0.6 & 1.5 \\
\hline $170 \mathrm{R}-3,41-43$ & 1255 & Sheeted dike & Aphyric & Microcrystalline & Hypocrystalline & Intersertal-subophitic & 0.0 & 0.0 & 0.0 \\
\hline
\end{tabular}

Notes: $\mathrm{CSF}=$ core depth below seafloor. Aphyric $=0 \%-1 \%$, sparsely phyric $=1 \%-5 \%$, moderately phyric $=5 \%-10 \%$. Cryptocrystalline $=<0.1$ $\mathrm{mm}$, microcrystalline $=0.1-0.2 \mathrm{~mm}$, fine grained $=0.2-1.0 \mathrm{~mm}$. 
Table T2. Average mineral compositions (in weight percent) of selected samples, Hole 1256D. (See table notes.) (Continued on next three pages.)

\begin{tabular}{|c|c|c|c|c|c|c|c|c|c|c|c|c|c|c|c|c|c|}
\hline Core, section, interval $(\mathrm{cm})$ & Lithology & Phase & $\begin{array}{l}\text { Analytical } \\
\text { method }\end{array}$ & Number & $\mathrm{SiO}_{2}$ & $\mathrm{TiO}_{2}$ & $\mathrm{Al}_{2} \mathrm{O}_{3}$ & $\mathrm{Cr}_{2} \mathrm{O}_{3}$ & $\mathrm{FeO}^{\text {tot }}$ & $\mathrm{MnO}$ & $\mathrm{MgO}$ & $\mathrm{CaO}$ & $\mathrm{Na}_{2} \mathrm{O}$ & $\mathrm{K}_{2} \mathrm{O}$ & Total & Mg\# & An \\
\hline \multirow{14}{*}{ 206-1256D-9R-2, 7-10 } & \multirow{14}{*}{ Ponded lava flow } & \multirow[t]{2}{*}{$\mathrm{cpx}$} & \multirow[t]{2}{*}{ Core } & \multirow[t]{2}{*}{8} & 52.65 & 0.52 & 1.85 & \multirow[t]{2}{*}{-} & 8.96 & 0.25 & 17.18 & 18.51 & 0.27 & \multirow[t]{2}{*}{-} & \multirow[t]{2}{*}{100.33} & 77.36 & \\
\hline & & & & & 0.76 & 0.06 & 0.20 & & 0.69 & 0.02 & 0.51 & 0.63 & 0.14 & & & 1.59 & \\
\hline & & \multirow[t]{2}{*}{$\mathrm{cpx}$} & \multirow[t]{4}{*}{ Rim } & \multirow[t]{2}{*}{18} & 51.31 & 0.72 & 1.41 & \multirow{2}{*}{-} & 16.37 & 0.43 & 14.89 & 14.91 & 0.23 & \multirow[t]{2}{*}{-} & \multirow{2}{*}{100.27} & 61.85 & \\
\hline & & & & & 0.52 & 0.08 & 0.21 & & 1.75 & 0.06 & 1.33 & 1.47 & 0.03 & & & 4.08 & \\
\hline & & \multirow{2}{*}{ pgt } & & \multirow{2}{*}{17} & 53.27 & 0.37 & 0.88 & - & 18.95 & 0.49 & 21.90 & 4.82 & 0.08 & - & 100.77 & 67.32 & \\
\hline & & & & & 0.95 & 0.09 & 0.20 & & 2.78 & 0.08 & 2.31 & 1.03 & 0.02 & & 0.58 & 5.53 & \\
\hline & & $\mathrm{pl}$ & Core & 5 & 53.21 & & 28.78 & & 0.75 & & 0.16 & 12.48 & 4.50 & 0.05 & 99.93 & & 60.36 \\
\hline & & & & & 2.02 & & 1.26 & & 0.11 & & 0.06 & 1.51 & 0.85 & 0.02 & & & 7.37 \\
\hline & & $\mathrm{pl}$ & $\operatorname{Rim}$ & 5 & 59.92 & & 25.10 & & 0.48 & & - & 7.25 & 7.43 & 0.15 & 100.33 & & 34.74 \\
\hline & & & & & 1.92 & & 1.04 & & 0.12 & & & 1.59 & 0.75 & 0.05 & & & 7.29 \\
\hline & & ilm & Focused beam & 10 & - & 49.36 & 0.04 & - & 47.90 & 0.54 & 0.43 & - & - & - & 98.35 & & \\
\hline & & & & & & 0.35 & 0.01 & & 0.32 & 0.07 & 0.06 & & & & & & \\
\hline & & mag & Focused beam & 21 & 0.14 & 20.96 & 0.86 & 0.05 & 71.75 & 1.00 & 0.30 & - & - & - & 95.08 & & \\
\hline & & & & & 0.12 & 1.22 & 0.09 & 0.02 & 1.43 & 0.51 & 0.04 & & & & & & \\
\hline 206-1256D-13R-1, 109-111 & Massive or sheet flow & $\operatorname{cpx}$ & Core & 13 & 51.22 & 0.97 & 3.27 & 0.23 & 7.97 & 0.20 & 17.69 & 18.73 & 0.23 & - & 100.52 & 79.81 & \\
\hline & & & & & 1.49 & 0.40 & 1.20 & 0.15 & 0.69 & 0.05 & 1.66 & 1.54 & 0.06 & & & 1.96 & \\
\hline & & $\operatorname{cpx}$ & $\operatorname{Rim}$ & 6 & 49.34 & 1.52 & 3.24 & - & 13.36 & 0.35 & 13.91 & 18.12 & 0.33 & - & 100.17 & 64.98 & \\
\hline & & & & & 0.52 & 0.13 & 0.30 & & 1.67 & 0.06 & 1.32 & 0.75 & 0.02 & & & 4.90 & \\
\hline & & $\mathrm{pl}$ & Core & 9 & 51.30 & & 30.23 & & 0.65 & & 0.24 & 14.06 & 3.65 & 0.06 & 100.18 & & 67.83 \\
\hline & & & & & 0.43 & & 0.26 & & 0.08 & & 0.02 & 0.22 & 0.14 & 0.01 & & & 1.15 \\
\hline & & $\mathrm{pl}$ & $\operatorname{Rim}$ & 4 & 57.23 & & 26.46 & & 0.78 & & 0.11 & 9.32 & 6.22 & 0.09 & 100.20 & & 45.07 \\
\hline & & & & & 2.59 & & 1.58 & & 0.11 & & 0.03 & 2.01 & 1.01 & 0.06 & & & 9.28 \\
\hline & & mag & Focused beam & 10 & 0.14 & 22.10 & 1.96 & - & 68.91 & 0.61 & 0.77 & 0.14 & - & - & 94.64 & & \\
\hline & & & & & 0.05 & 0.41 & 0.14 & & 0.52 & 0.05 & 0.12 & 0.03 & & & & & \\
\hline 206-1256D-32R-2, 6-8 & Massive or sheet flow & $\mathrm{cpx}$ & Core & 5 & 52.06 & 0.53 & 1.94 & - & 12.12 & 0.32 & 18.61 & 14.37 & 0.15 & - & 100.14 & 73.24 & \\
\hline & & & & & 0.50 & 0.12 & 0.62 & & 2.72 & 0.06 & 1.11 & 2.88 & 0.03 & & & 3.57 & \\
\hline & & cpx & $\operatorname{Rim}$ & 3 & 48.59 & 0.87 & 1.33 & - & 24.63 & 0.52 & 10.02 & 13.20 & 0.16 & - & 99.36 & 42.02 & \\
\hline & & & & & 0.60 & 0.09 & 0.19 & & 3.04 & 0.08 & 1.42 & 1.80 & 0.03 & & & 6.25 & \\
\hline & & pgt & & 5 & 54.57 & 0.20 & 0.74 & - & 13.38 & 0.37 & 26.07 & 5.13 & - & - & 100.56 & 77.65 & \\
\hline & & & & & 0.40 & 0.05 & 0.13 & & 1.11 & 0.04 & 1.46 & 1.98 & & & 0.36 & 1.53 & \\
\hline & & $\mathrm{pl}$ & Core & 11 & 51.99 & & 29.36 & & 0.83 & & 0.23 & 13.47 & 3.91 & - & 99.83 & & 65.59 \\
\hline & & & & & 0.46 & & 0.42 & & 0.10 & & 0.03 & 0.31 & 0.23 & & & & 1.78 \\
\hline & & $\mathrm{pl}$ & $\operatorname{Rim}$ & 3 & 56.86 & & 25.85 & & 1.25 & & 0.14 & 9.56 & 6.08 & 0.08 & 99.82 & & 46.28 \\
\hline & & & & & 1.14 & & 0.28 & & 0.60 & & 0.16 & 0.27 & 0.37 & 0.00 & & & 2.22 \\
\hline & & mag & Focused beam & 11 & 0.17 & 20.71 & 1.89 & - & 71.93 & 0.48 & 0.21 & 0.05 & - & - & 95.50 & & \\
\hline & & & & & 0.05 & 0.39 & 0.20 & & 0.39 & 0.07 & 0.05 & 0.03 & & & & & \\
\hline 206-1256D-39R-2, 9-10 & Massive or sheet flow & $\operatorname{cpx}$ & Core & 5 & 52.62 & 0.37 & 2.77 & 0.44 & 6.70 & 0.17 & 19.07 & 18.24 & 0.18 & - & 100.56 & 83.54 & \\
\hline & & & & & 0.42 & 0.09 & 0.31 & 0.21 & 0.51 & 0.05 & 1.06 & 1.34 & 0.02 & & & 1.13 & \\
\hline & & $\operatorname{cpx}$ & $\operatorname{Rim}$ & 3 & 49.33 & 0.78 & 1.38 & - & 20.34 & 0.47 & 12.13 & 14.47 & 0.18 & - & 99.11 & 51.52 & \\
\hline & & & & & 0.59 & 0.17 & 0.46 & & 1.92 & 0.01 & 0.21 & 1.64 & 0.04 & & & 2.60 & \\
\hline & & $\operatorname{cpx}$ & Core* & 5 & 53.10 & 0.23 & 1.94 & 0.49 & 5.90 & 0.18 & 19.61 & 18.60 & 0.17 & - & 100.23 & 85.55 & \\
\hline & & & & & 0.31 & 0.03 & 0.29 & 0.06 & 0.25 & 0.02 & 0.49 & 0.51 & 0.01 & & & 0.28 & \\
\hline & & pgt & & 5 & 55.17 & 0.11 & 0.70 & - & 11.01 & 0.29 & 28.38 & 4.39 & - & - & 100.17 & 82.12 & \\
\hline & & & & & 0.28 & 0.01 & 0.06 & & 0.40 & 0.04 & 0.13 & 0.19 & & & 0.42 & 0.54 & \\
\hline & & $\mathrm{pl}$ & Core & 18 & 51.68 & & 29.25 & & 0.94 & & 0.30 & 13.54 & 3.76 & 0.04 & 99.52 & & 66.39 \\
\hline & & & & & 1.16 & & 0.94 & & 0.39 & & 0.19 & 0.99 & 0.57 & 0.01 & & & 4.85 \\
\hline & & $\mathrm{pl}$ & $\operatorname{Rim}$ & 4 & 55.52 & & 26.49 & & 1.17 & & 0.13 & 10.65 & 5.33 & 0.08 & 99.36 & & 52.22 \\
\hline & & & & & 1.96 & & 1.10 & & 0.34 & & 0.18 & 1.24 & 0.76 & 0.02 & & & 6.48 \\
\hline
\end{tabular}

mag Focused beam

$\begin{array}{lll}0.14 & 20.09 & 2\end{array}$


Table T2 (continued). (Continued on next page.)

\begin{tabular}{|c|c|c|c|c|c|c|c|c|c|c|c|c|c|c|c|c|c|}
\hline Core, section, interval $(\mathrm{cm})$ & Lithology & Phase & $\begin{array}{l}\text { Analytical } \\
\text { method }\end{array}$ & Number & $\mathrm{SiO}_{2}$ & $\mathrm{TiO}_{2}$ & $\mathrm{Al}_{2} \mathrm{O}_{3}$ & $\mathrm{Cr}_{2} \mathrm{O}_{3}$ & $\mathrm{FeO}^{\text {tot }}$ & $\mathrm{MnO}$ & $\mathrm{MgO}$ & $\mathrm{CaO}$ & $\mathrm{Na}_{2} \mathrm{O}$ & $\mathrm{K}_{2} \mathrm{O}$ & Total & Mg\# & An \\
\hline & & & & & 0.02 & 0.36 & 0.08 & & 0.33 & 0.05 & 0.07 & 0.02 & & & & & \\
\hline \multirow[t]{12}{*}{ 206-1256D-45R-1, 92-95 } & Massive or sheet flow & $\mathrm{cpx}$ & Core & 7 & 50.19 & 1.01 & 5.18 & 0.24 & 7.39 & 0.18 & 16.67 & 18.97 & 0.19 & - & 100.02 & 80.09 & \\
\hline & & & & & 0.49 & 0.09 & 1.04 & 0.15 & 0.96 & 0.03 & 0.72 & 1.01 & 0.02 & & & 2.34 & \\
\hline & & cpx & Rim & 3 & 49.36 & 1.23 & 3.50 & - & 12.59 & 0.30 & 13.51 & 19.08 & 0.23 & - & 99.82 & 65.66 & \\
\hline & & & & & 0.90 & 0.28 & 0.34 & & 2.72 & 0.09 & 2.32 & 0.98 & 0.02 & & & 8.86 & \\
\hline & & $\mathrm{pl}$ & Core & 4 & 52.10 & & 29.42 & & 1.02 & & 0.22 & 13.60 & 3.84 & 0.13 & 100.33 & & 65.66 \\
\hline & & & & & 0.21 & & 0.19 & & 0.05 & & 0.06 & 0.23 & 0.13 & 0.01 & & & 1.11 \\
\hline & & $\mathrm{pl}$ & Rim & 3 & 54.00 & & 28.10 & & 1.15 & & 0.12 & 11.89 & 4.75 & 0.21 & 100.22 & & 57.33 \\
\hline & & & & & 0.39 & & 0.17 & & 0.07 & & 0.01 & 0.29 & 0.15 & 0.03 & & & 1.34 \\
\hline & & $\mathrm{pl}$ & Core $^{*}$ & 5 & 47.30 & & 32.89 & & 0.49 & & 0.23 & 17.21 & 1.82 & 0.04 & 99.99 & & 83.72 \\
\hline & & & & & 1.25 & & 0.81 & & 0.14 & & 0.04 & 0.95 & 0.45 & 0.01 & & & 4.09 \\
\hline & & $\mathrm{pl}$ & $\operatorname{Rim}^{*}$ & 4 & 50.93 & & 30.00 & & 0.73 & & 0.30 & 14.63 & 3.26 & 0.08 & 99.95 & & 70.91 \\
\hline & & & & & 0.70 & & 0.43 & & 0.13 & & 0.03 & 0.51 & 0.30 & 0.02 & & & 2.55 \\
\hline \multirow[t]{12}{*}{ 206-1256D-49R-2, 38-40 } & Massive or sheet flow & cpx & Core & 13 & 52.18 & 0.53 & 2.73 & 0.36 & 6.94 & 0.21 & 19.57 & 17.32 & 0.19 & 0.01 & 100.05 & 83.40 & \\
\hline & & & & & 1.32 & 0.24 & 1.22 & 0.26 & 0.79 & 0.04 & 1.89 & 2.14 & 0.04 & 0.01 & & 1.52 & \\
\hline & & cpx & Rim & 2 & 48.90 & 1.19 & 2.73 & - & 16.18 & 0.35 & 13.86 & 15.16 & 0.29 & - & 98.74 & 60.42 & \\
\hline & & & & & 1.15 & 0.08 & 0.48 & & 4.12 & 0.10 & 3.03 & 0.20 & 0.05 & & & 11.33 & \\
\hline & & $\mathrm{pl}$ & Core & 6 & 51.04 & & 29.55 & & 0.79 & & 0.38 & 14.17 & 3.47 & 0.06 & 99.46 & & 69.06 \\
\hline & & & & & 0.80 & & 0.46 & & 0.26 & & 0.28 & 0.40 & 0.36 & 0.08 & & & 2.68 \\
\hline & & $\mathrm{pl}$ & Rim & 5 & 51.52 & & 29.26 & & 0.80 & & 0.28 & 13.82 & 3.71 & 0.04 & 99.44 & & 67.13 \\
\hline & & & & & 0.71 & & 0.56 & & 0.09 & & 0.05 & 0.59 & 0.32 & 0.01 & & & 2.82 \\
\hline & & $\mathrm{pl}$ & Core* & 5 & 48.26 & & 31.61 & & 0.46 & & 0.23 & 16.23 & 2.32 & - & 99.14 & & 79.44 \\
\hline & & & & & 0.98 & & 0.63 & & 0.04 & & 0.04 & 0.65 & 0.36 & & & & 3.16 \\
\hline & & $\mathrm{pl}$ & $\mathrm{Rim}^{*}$ & 5 & 53.50 & & 28.06 & & 0.87 & & 0.22 & 12.23 & 4.59 & 0.06 & 99.53 & & 59.35 \\
\hline & & & & & 3.26 & & 2.45 & & 0.22 & & 0.15 & 2.67 & 1.50 & 0.04 & & & 13.17 \\
\hline \multirow[t]{12}{*}{ 206-1256D-52R-1, 5-7 } & Massive or sheet flow & cpx & Core & 7 & 51.06 & 0.82 & 3.26 & - & 10.55 & 0.27 & 18.49 & 15.35 & 0.21 & - & 100.06 & 75.74 & \\
\hline & & & & & 0.66 & 0.15 & 0.41 & & 0.87 & 0.04 & 1.45 & 1.78 & 0.03 & & & 1.66 & \\
\hline & & $c p x$ & Rim & 4 & 49.42 & 0.93 & 1.66 & - & 20.12 & 0.48 & 11.46 & 15.23 & 0.21 & - & 99.52 & 50.38 & \\
\hline & & & & & 0.64 & 0.29 & 0.56 & & 2.70 & 0.10 & 1.61 & 3.39 & 0.06 & & & 3.87 & \\
\hline & & pgt & & 6 & 54.19 & 0.25 & 1.05 & - & 14.26 & 0.37 & 26.34 & 4.58 & - & - & 101.13 & 76.70 & \\
\hline & & & & & 0.90 & 0.08 & 0.43 & & 2.66 & 0.06 & 2.21 & 0.99 & & & 0.43 & 4.77 & \\
\hline & & $\mathrm{pl}$ & Core & 10 & 54.41 & & 27.60 & & 1.12 & & 0.14 & 11.41 & 4.99 & 0.06 & 99.73 & & 55.62 \\
\hline & & & & & 0.67 & & 0.46 & & 0.14 & & 0.05 & 0.61 & 0.36 & 0.01 & & & 3.05 \\
\hline & & $\mathrm{pl}$ & Rim & 6 & 59.64 & & 24.72 & & 0.97 & & - & 7.72 & 6.90 & 0.12 & 100.09 & & 37.91 \\
\hline & & & & & 2.29 & & 1.13 & & 0.16 & & & 1.63 & 0.81 & 0.06 & & & 7.83 \\
\hline & & mag & Focused beam & 5 & 0.21 & 21.79 & 1.96 & - & 67.83 & 0.78 & 0.18 & 0.11 & - & - & 92.93 & & \\
\hline & & & & & 0.16 & 0.43 & 0.13 & & 0.50 & 0.13 & 0.04 & 0.04 & & & & & \\
\hline \multirow{8}{*}{ 206-1256D-57R-2, 24-26 } & Massive or sheet flow & $\mathrm{cpx}$ & Core & 4 & 52.66 & 0.45 & 1.99 & 0.22 & 9.30 & 0.27 & 19.15 & 16.28 & 0.17 & - & 100.49 & 78.59 & \\
\hline & & & & & 0.71 & 0.15 & 0.86 & 0.33 & 2.32 & 0.04 & 1.51 & 2.79 & 0.04 & & & 3.98 & \\
\hline & & $\mathrm{pl}$ & Core & 15 & 52.55 & & 29.03 & & 0.80 & & 0.20 & 13.03 & 4.22 & 0.05 & 99.88 & & 62.86 \\
\hline & & & & & 0.76 & & 0.45 & & 0.12 & & 0.04 & 0.67 & 0.36 & 0.01 & & & 3.17 \\
\hline & & $\mathrm{pl}$ & $\operatorname{Rim}$ & 5 & 57.80 & & 25.89 & & 0.73 & & - & 9.08 & 6.45 & 0.08 & 100.04 & & 43.54 \\
\hline & & & & & 0.70 & & 0.23 & & 0.09 & & & 0.36 & 0.19 & 0.00 & & & 1.66 \\
\hline & & mag & Focused beam & 15 & 0.30 & 22.31 & 1.70 & - & 70.23 & 0.95 & 0.17 & 0.05 & - & - & 95.76 & & \\
\hline & & & & & 0.55 & 0.79 & 0.20 & & 1.23 & 0.22 & 0.10 & 0.05 & & & & & \\
\hline \multirow[t]{5}{*}{ 309-1256D-76R-2, 0-4 } & Massive or sheet flow & $\mathrm{cpx}$ & Core & 7 & 51.95 & 0.59 & 2.61 & - & 10.29 & 0.27 & 18.88 & 15.56 & 0.18 & - & 100.38 & 76.57 & \\
\hline & & & & & 0.71 & 0.10 & 0.35 & & 1.00 & 0.05 & 1.42 & 1.90 & 0.04 & & & 1.99 & \\
\hline & & cpx & $\operatorname{Rim}$ & 5 & 48.95 & 1.14 & 2.11 & - & 19.69 & 0.44 & 12.32 & 14.61 & 0.27 & - & 99.56 & 52.73 & \\
\hline & & & & & 0.95 & 0.33 & 0.69 & & 3.36 & 0.08 & 1.74 & 3.47 & 0.12 & & & 5.16 & \\
\hline & & cpx & Core $^{*}$ & 12 & 52.98 & 0.37 & 2.11 & - & 8.16 & 0.24 & 19.52 & 16.81 & 0.18 & - & 100.50 & 81.00 & \\
\hline
\end{tabular}


Table T2 (continued). (Continued on next page.)

\begin{tabular}{|c|c|c|c|c|c|c|c|c|c|c|c|c|c|c|c|c|c|}
\hline Core, section, interval $(\mathrm{cm})$ & Lithology & Phase & $\begin{array}{l}\text { Analytical } \\
\text { method }\end{array}$ & Number & $\mathrm{SiO}_{2}$ & $\mathrm{TiO}_{2}$ & $\mathrm{Al}_{2} \mathrm{O}_{3}$ & $\mathrm{Cr}_{2} \mathrm{O}_{3}$ & $\mathrm{FeO}^{\text {tot }}$ & $\mathrm{MnO}$ & $\mathrm{MgO}$ & $\mathrm{CaO}$ & $\mathrm{Na}_{2} \mathrm{O}$ & $\mathrm{K}_{2} \mathrm{O}$ & Total & Mg\# & An \\
\hline & & pgt & & 3 & 54.85 & 0.19 & 1.00 & - & 12.56 & 0.34 & 26.78 & 5.17 & 0.06 & - & 101.01 & 79.17 & \\
\hline & & & & & 0.17 & 0.01 & 0.35 & & 0.25 & 0.06 & 0.87 & 1.25 & 0.03 & & & 0.33 & \\
\hline & & $\mathrm{pl}$ & Core & 6 & 53.99 & & 27.82 & & 1.03 & & 0.16 & 11.99 & 4.76 & 0.05 & 99.81 & & 57.99 \\
\hline & & & & & 1.11 & & 0.87 & & 0.18 & & 0.05 & 0.96 & 0.53 & 0.01 & & & 4.66 \\
\hline & & $\mathrm{pl}$ & $\operatorname{Rim}^{*}$ & 13 & 52.66 & & 28.87 & & 0.81 & & 0.21 & 13.14 & 4.13 & 0.04 & 99.86 & & 63.58 \\
\hline & & & & & 1.48 & & 1.12 & & 0.23 & & 0.03 & 1.17 & 0.66 & 0.01 & & & 5.73 \\
\hline & & mag & Focused beam & 5 & 0.20 & 22.14 & 2.25 & - & 67.40 & 1.70 & - & 0.23 & - & - & 93.99 & & \\
\hline & & & & & 0.04 & 0.21 & 0.21 & & 1.14 & 0.11 & & 0.06 & & & & & \\
\hline \multirow[t]{10}{*}{ 309-1256D-85R-3, 76-78 } & Massive or sheet flow & $\mathrm{cpx}$ & Core & 7 & 52.45 & 0.48 & 2.46 & - & 8.32 & 0.25 & 17.98 & 17.31 & 0.18 & - & 99.59 & 79.39 & \\
\hline & & & & & 1.12 & 0.20 & 1.07 & & 0.68 & 0.04 & 1.58 & 1.66 & 0.05 & & & 1.60 & \\
\hline & & $\mathrm{cpx}$ & Rim & 3 & 49.43 & 0.93 & 2.09 & - & 18.07 & 0.40 & 11.93 & 15.78 & 0.23 & - & 98.87 & 54.05 & \\
\hline & & & & & 0.89 & 0.09 & 0.49 & & 4.46 & 0.13 & 2.94 & 0.39 & 0.05 & & & 12.32 & \\
\hline & & $\mathrm{pl}$ & Core & 12 & 51.01 & & 29.85 & & 0.69 & & 0.27 & 14.02 & 3.57 & - & 99.44 & & 68.43 \\
\hline & & & & & 1.32 & & 0.83 & & 0.07 & & 0.07 & 0.84 & 0.50 & & & & 4.29 \\
\hline & & $\mathrm{pl}$ & $\operatorname{Rim}$ & 12 & 54.30 & & 27.62 & & 0.93 & & 0.15 & 11.44 & 5.01 & 0.05 & 99.51 & & 55.61 \\
\hline & & & & & 2.11 & & 1.38 & & 0.15 & & 0.08 & 1.60 & 0.88 & 0.02 & & & 7.79 \\
\hline & & mag & Focused beam & 9 & 0.14 & 21.45 & 2.07 & - & 68.95 & 1.73 & - & 0.08 & - & - & 94.49 & & \\
\hline & & & & & 0.02 & 0.75 & 0.21 & & 0.71 & 0.08 & & 0.03 & & & & & \\
\hline \multirow{10}{*}{ 309-1256D-87R-2, 63-66 } & Massive or sheet flow & $\mathrm{cpx}$ & Core & 13 & 52.15 & 0.49 & 2.43 & - & 7.88 & 0.22 & 18.03 & 18.83 & 0.20 & - & 100.38 & 80.31 & \\
\hline & & & & & 0.60 & 0.11 & 0.60 & & 0.74 & 0.03 & 0.83 & 0.80 & 0.02 & & & 1.90 & \\
\hline & & $\operatorname{cpx}$ & $\operatorname{Rim}$ & 3 & 49.99 & 0.78 & 1.72 & - & 17.78 & 0.43 & 13.78 & 15.25 & 0.22 & - & 99.96 & 58.01 & \\
\hline & & & & & 0.76 & 0.18 & 0.07 & & 1.23 & 0.04 & 3.67 & 4.10 & 0.07 & & & 7.18 & \\
\hline & & $\mathrm{pl}$ & Core & 14 & 52.46 & & 28.54 & & 0.85 & & 0.29 & 13.05 & 4.22 & 0.04 & 99.44 & & 62.95 \\
\hline & & & & & 1.81 & & 1.38 & & 0.30 & & 0.28 & 1.43 & 0.75 & 0.01 & & & 6.65 \\
\hline & & $\mathrm{pl}$ & $\operatorname{Rim}$ & 5 & 58.19 & & 25.59 & & 0.74 & & - & 8.93 & 6.54 & 0.06 & 100.08 & & 42.86 \\
\hline & & & & & 1.42 & & 0.86 & & 0.10 & & & 1.09 & 0.59 & 0.01 & & & 5.18 \\
\hline & & mag & Focused beam & 11 & 0.15 & 22.60 & 1.82 & - & 68.76 & 1.71 & - & 0.07 & - & - & 95.17 & & \\
\hline & & & & & 0.01 & 0.46 & 0.28 & & 0.72 & 0.07 & & 0.02 & & & & & \\
\hline \multirow[t]{14}{*}{ 309-1256D-102R-1, 70-72 } & Massive or sheet flow & $\mathrm{cpx}$ & Core & 7 & 50.70 & 0.77 & 3.63 & 0.27 & 8.01 & 0.17 & 16.95 & 18.80 & 0.21 & - & 99.51 & 79.04 & \\
\hline & & & & & 0.66 & 0.14 & 0.36 & 0.07 & 0.60 & 0.03 & 0.93 & 0.89 & 0.02 & & & 1.97 & \\
\hline & & $\mathrm{cpx}$ & Rim & 10 & 49.43 & 1.02 & 2.28 & - & 18.33 & 0.42 & 14.18 & 13.57 & 0.19 & - & 99.45 & 57.96 & \\
\hline & & & & & 1.54 & 0.36 & 0.34 & & 4.87 & 0.11 & 3.81 & 2.63 & 0.03 & & & 12.92 & \\
\hline & & pl & Core & 11 & 51.44 & & 29.58 & & 0.75 & & 0.27 & 13.96 & 3.57 & - & 99.60 & & 68.35 \\
\hline & & & & & 0.67 & & 0.55 & & 0.11 & & 0.04 & 0.52 & 0.29 & & & & 2.53 \\
\hline & & $\mathrm{pl}$ & $\operatorname{Rim}$ & 7 & 56.28 & & 26.30 & & 1.02 & & 0.08 & 10.18 & 5.63 & 0.07 & 99.56 & & 49.76 \\
\hline & & & & & 1.03 & & 0.68 & & 0.11 & & 0.03 & 0.80 & 0.42 & 0.01 & & & 3.80 \\
\hline & & pl & Core* & 6 & 50.54 & & 30.00 & & 0.70 & & 0.36 & 14.70 & 3.18 & - & 99.51 & & 71.86 \\
\hline & & & & & 0.82 & & 0.84 & & 0.25 & & 0.20 & 0.70 & 0.29 & & & & 2.80 \\
\hline & & pl & $\operatorname{Rim}^{*}$ & 6 & 53.47 & & 28.05 & & 0.97 & & 0.20 & 12.28 & 4.50 & 0.04 & 99.51 & & 59.97 \\
\hline & & & & & 2.22 & & 1.60 & & 0.25 & & 0.05 & 1.91 & 0.98 & 0.02 & & & 9.02 \\
\hline & & mag & Focused beam & 10 & 0.14 & 20.59 & 2.23 & - & 69.87 & 1.18 & - & 0.08 & - & - & 94.15 & & \\
\hline & & & & & 0.02 & 1.00 & 0.29 & & 1.04 & 0.07 & & 0.04 & & & & & \\
\hline \multirow[t]{8}{*}{ 309-1256D-118R-1, 43-48 } & Massive or sheet flow & $\mathrm{cpx}$ & Core & 7 & 52.84 & 0.37 & 2.08 & 0.35 & 8.25 & 0.23 & 20.00 & 16.29 & 0.16 & - & 100.58 & 81.21 & \\
\hline & & & & & 1.01 & 0.13 & 0.71 & 0.15 & 0.69 & 0.06 & 1.74 & 2.11 & 0.03 & & & 1.24 & \\
\hline & & $\mathrm{cpx}$ & Rim & 1 & 48.83 & 0.77 & 1.56 & - & 19.03 & 0.48 & 11.46 & 16.45 & 0.22 & - & 98.82 & 51.75 & \\
\hline & & $\mathrm{cpx}$ & Core* & 7 & 51.77 & 0.36 & 3.04 & 0.97 & 7.31 & 0.24 & 18.10 & 18.26 & 0.22 & - & 100.27 & 81.51 & \\
\hline & & & & & 0.73 & 0.05 & 0.23 & 0.11 & 0.90 & 0.06 & 2.63 & 2.15 & 0.05 & & & 3.99 & \\
\hline & & $\mathrm{pl}$ & Core & 9 & 51.01 & & 29.66 & & 0.68 & & 0.24 & 14.20 & 3.45 & - & 99.27 & & 69.47 \\
\hline & & & & & 0.99 & & 0.58 & & 0.15 & & 0.04 & 0.71 & 0.42 & & & & 3.60 \\
\hline & & $\mathrm{pl}$ & Rim & 4 & 56.90 & & 26.16 & & 0.79 & & 0.04 & 9.67 & 5.91 & 0.10 & 99.57 & & 47.20 \\
\hline
\end{tabular}


Table T2 (continued).

\begin{tabular}{|c|c|c|c|c|c|c|c|c|c|c|c|c|c|c|c|c|c|}
\hline Core, section, interval $(\mathrm{cm})$ & Lithology & Phase & $\begin{array}{l}\text { Analytical } \\
\text { method }\end{array}$ & Number & $\mathrm{SiO}_{2}$ & $\mathrm{TiO}_{2}$ & $\mathrm{Al}_{2} \mathrm{O}_{3}$ & $\mathrm{Cr}_{2} \mathrm{O}_{3}$ & $\mathrm{FeO}^{\text {tot }}$ & $\mathrm{MnO}$ & $\mathrm{MgO}$ & $\mathrm{CaO}$ & $\mathrm{Na}_{2} \mathrm{O}$ & $\mathrm{K}_{2} \mathrm{O}$ & Total & Mg\# & An \\
\hline & & & & & 1.30 & & 0.70 & & 0.21 & & 0.03 & 1.04 & 0.67 & 0.04 & & & 5.46 \\
\hline & & $\mathrm{pl}$ & Core $^{*}$ & 7 & 49.32 & & 31.30 & & 0.52 & & 0.26 & 15.60 & 2.75 & - & 99.77 & & 75.84 \\
\hline & & & & & 0.55 & & 0.36 & & 0.05 & & 0.04 & 0.43 & 0.22 & & & & 1.91 \\
\hline & & $\mathrm{pl}$ & $\operatorname{Rim}^{*}$ & 4 & 55.05 & & 27.02 & & 0.81 & & 0.07 & 10.77 & 5.44 & 0.07 & 99.22 & & 52.04 \\
\hline & & & & & 0.51 & & 0.63 & & 0.06 & & 0.02 & 0.36 & 0.21 & 0.01 & & & 1.81 \\
\hline & & mag & Focused beam & 7 & 0.69 & 21.25 & 2.01 & - & 67.27 & 2.50 & 0.18 & 0.21 & - & - & 94.19 & & \\
\hline & & & & & 0.90 & 1.31 & 0.33 & & 0.62 & 0.17 & 0.36 & 0.11 & & & & & \\
\hline \multirow[t]{8}{*}{ 309-1256D-147R-1, 71-75 } & Sheeted dike & $\mathrm{cpx}$ & Core & 13 & 51.93 & 0.52 & 2.16 & - & 10.00 & 0.28 & 18.05 & 16.73 & 0.20 & - & 100.02 & 76.29 & \\
\hline & & & & & 0.89 & 0.14 & 0.60 & & 2.15 & 0.05 & 1.21 & 2.28 & 0.03 & & & 3.88 & \\
\hline & & $\mathrm{cpx}$ & $\operatorname{Rim}$ & 6 & 50.52 & 0.60 & 1.54 & - & 15.67 & 0.38 & 13.69 & 16.73 & 0.22 & - & 99.38 & 60.88 & \\
\hline & & & & & 1.28 & 0.26 & 0.52 & & 5.05 & 0.11 & 4.53 & 1.53 & 0.03 & & & 16.19 & \\
\hline & & $\mathrm{pl}$ & Core & 8 & 52.89 & & 28.69 & & 0.72 & & 0.15 & 12.71 & 4.33 & 0.04 & 99.52 & & 61.73 \\
\hline & & & & & 1.02 & & 0.73 & & 0.09 & & 0.04 & 0.88 & 0.50 & 0.01 & & & 4.35 \\
\hline & & mag & Broad beam & 2 & 0.67 & 23.87 & 1.77 & - & 65.14 & 1.24 & 0.09 & 0.55 & - & - & 93.39 & & \\
\hline & & & & & 0.79 & 0.87 & 0.28 & & 1.01 & 0.05 & 0.12 & 0.47 & & & & & \\
\hline \multirow[t]{10}{*}{ 309-1256D-154R-1, 56-60 } & Sheeted dike & $\mathrm{cpx}$ & Core & 11 & 50.85 & 0.80 & 2.69 & - & 11.21 & 0.28 & 16.37 & 17.65 & 0.22 & - & 100.15 & 72.24 & \\
\hline & & & & & 0.51 & 0.11 & 0.22 & & 1.83 & 0.05 & 1.36 & 1.00 & 0.03 & & & 4.75 & \\
\hline & & $\mathrm{cpx}$ & $\operatorname{Rim}$ & 4 & 48.99 & 0.93 & 1.60 & - & 20.88 & 0.51 & 9.62 & 16.96 & 0.22 & - & 99.75 & 45.08 & \\
\hline & & & & & 0.52 & 0.11 & 0.24 & & 2.01 & 0.04 & 2.18 & 0.78 & 0.03 & & & 7.76 & \\
\hline & & $\mathrm{pl}$ & Core & 8 & 54.43 & & 27.69 & & 0.97 & & 0.12 & 11.53 & 5.06 & 0.06 & 99.85 & & 55.54 \\
\hline & & & & & 1.37 & & 0.82 & & 0.16 & & 0.05 & 1.00 & 0.51 & 0.01 & & & 4.65 \\
\hline & & $\mathrm{pl}$ & $\operatorname{Rim}$ & 8 & 56.80 & & 26.18 & & 1.05 & & 0.09 & 9.63 & 6.01 & 0.08 & 99.83 & & 46.76 \\
\hline & & & & & 2.36 & & 1.23 & & 0.37 & & 0.11 & 1.62 & 0.83 & 0.02 & & & 7.61 \\
\hline & & mag & Focused beam & 8 & 0.20 & 21.16 & 1.30 & - & 68.00 & 2.13 & - & 0.22 & - & - & 93.12 & & \\
\hline & & & & & 0.05 & 2.08 & 0.47 & & 2.81 & 0.44 & & 0.05 & & & & & \\
\hline \multirow{10}{*}{ 309-1256D-164R-2, 115-118 } & Sheeted dike & $\mathrm{cpx}$ & Core & 9 & 50.81 & 0.71 & 2.46 & - & 11.89 & 0.29 & 15.84 & 17.61 & 0.24 & - & 99.87 & 70.37 & \\
\hline & & & & & 0.37 & 0.10 & 0.25 & & 0.76 & 0.03 & 0.92 & 1.06 & 0.02 & & & 2.22 & \\
\hline & & $\mathrm{cpx}$ & $\operatorname{Rim}$ & 4 & 49.40 & 0.82 & 1.58 & - & 18.97 & 0.43 & 10.47 & 18.04 & 0.24 & - & 99.98 & 49.58 & \\
\hline & & & & & 0.66 & 0.05 & 0.08 & & 2.23 & 0.03 & 1.40 & 0.89 & 0.04 & & & 6.23 & \\
\hline & & $\mathrm{pl}$ & Core & 8 & 53.29 & & 28.20 & & 0.92 & & 0.16 & 12.04 & 4.71 & 0.05 & 99.36 & & 58.38 \\
\hline & & & & & 0.79 & & 0.52 & & 0.07 & & 0.03 & 0.63 & 0.33 & 0.01 & & & 2.97 \\
\hline & & $\mathrm{pl}$ & $\operatorname{Rim}$ & 6 & 58.22 & & 25.49 & & 0.75 & & - & 8.43 & 6.57 & 0.11 & 99.59 & & 41.22 \\
\hline & & & & & 2.72 & & 1.53 & & 0.21 & & & 1.85 & 0.93 & 0.07 & & & 8.86 \\
\hline & & mag & Focused beam & 12 & 1.03 & 24.08 & 1.10 & - & 65.68 & 0.95 & 0.13 & 0.75 & - & - & 93.79 & & \\
\hline & & & & & 1.14 & 1.35 & 0.18 & & 2.29 & 0.54 & 0.07 & 1.03 & & & & & \\
\hline
\end{tabular}

Notes: Number $=$ number of phases. One standard deviation is listed below its average composition in italics. $\mathrm{cpx}=$ clinopyroxene, $\mathrm{pgt}=$ pigeonite, $\mathrm{pl}=\mathrm{plagioclase}$ ilm $=\mathrm{ilmenite}$, mag $=$ magnetite. Broad beam analysis was used to reintegrate exsolution lamellae. ${ }^{*}=$ phenocryst. $A n=$ mol\% content of plagioclase. $-=$ below limit of detection, empty cell $=$ not analyzed. 